\title{
Accurate non-bonded potentials based on periodic quantum mechanics calculations for use in molecular simulations of materials and systems
}

Cite as: J. Chem. Phys. 151, 154111 (2019); https://doi.org/10.1063/1.5113811

Submitted: 09 June 2019. Accepted: 24 September 2019. Published Online: 18 October 2019

Saber Naserifar (D), Julius J. Oppenheim (D), Hao Yang, Tingting Zhou, Sergey Zybin, Mohamed Rizk, and William A. Goddard (D)
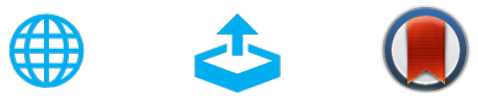

View Online

Export Citation

\section{ARTICLES YOU MAY BE INTERESTED IN}

In search of Coulson's lost theorem

The Journal of Chemical Physics 151, 151101 (2019); https://doi.org/10.1063/1.5128624

Force balance approach for advanced approximations in density functional theories The Journal of Chemical Physics 151, 154107 (2019); https://doi.org/10.1063/1.5123608

Speeding-up ab initio molecular dynamics with hybrid functionals using adaptively compressed exchange operator based multiple timestepping

The Journal of Chemical Physics 151, 151102 (2019); https://doi.org/10.1063/1.5125422

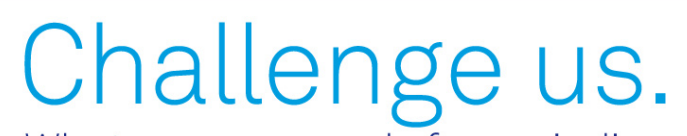

What are your needs for periodic signal detection?
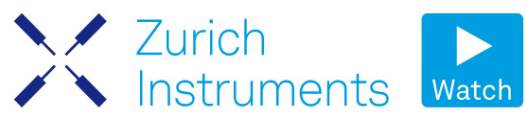

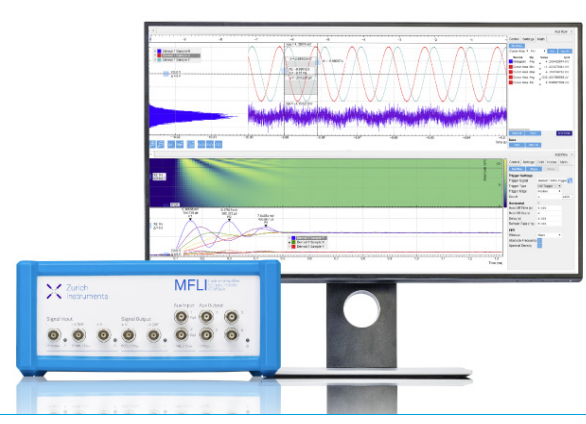

151, 154111 


\title{
Accurate non-bonded potentials based on periodic quantum mechanics calculations for use in molecular simulations of materials and systems
}

\author{
Cite as: J. Chem. Phys. 151, 154111 (2019); doi: 10.1063/1.5113811 \\ Submitted: 9 June 2019 Accepted: 24 September 2019 • \\ Published Online: 18 October 2019
}

Saber Naserifar, ${ }^{1}$ (D) Julius J. Oppenheim, ${ }^{1}$ (D) Hao Yang, ${ }^{1}$ Tingting Zhou, ${ }^{1,2}$ Sergey Zybin, ${ }^{1}$ Mohamed Rizk, and William A. Goddard III, (i)

\begin{abstract}
AFFILIATIONS
${ }^{1}$ Materials and Process Simulation Center, California Institute of Technology, Pasadena, California 91125, USA

${ }^{2}$ Institute of Applied Physics and Computational Mathematics, Fenghao Donglu, Haidian District, Beijing 100094, China
\end{abstract}

a) Author to whom correspondence should be addressed: wag@caltech.edu

\begin{abstract}
Molecular dynamics simulations require accurate force fields (FFs) to describe the physical and chemical properties of complex materials and systems. FF parameters for valence interactions can be determined from high-quality Quantum Mechanical (QM) calculations. However, it has been challenging to extract long-range nonbonded interaction potentials from QM calculations since there is no unambiguous method to separate the total QM energy into electrostatics (polarization), van der Waals (vdW), and other components. Here, we propose to use density functional theory with dispersion corrections to obtain the equation of state for single element solid systems (of $\mathrm{H}, \mathrm{C}, \mathrm{N}, \mathrm{O}, \mathrm{F}, \mathrm{Cl}, \mathrm{Br}$, I, P, He, Ne, Ar, Kr, Xe, and Rn) from which we obtain the pure 2-body vdW nonbonded potentials. Recently, we developed the polarizable charge equilibration (PQEq) model based on QM polarization energy of electric probe dipoles with no contributions from vdW. Together, the vdW and PQEq interactions form the nonbonded potential of our new transferrable reactive FF (RexPoN). They may also be useful to replace the nonbonded parts of standard FFs, such as OPLS, Amber, UFF, and CHARMM. We find that the individual 2-body vdW potential curves can be scaled to a universal vdW potential using just three specific atomic parameters. This simplifies extension to the rest of the periodic table for atoms that do not exhibit molecular packing. We validate the accuracy of these nonbonded interactions for liquid water, energetic, and biological systems. In all cases, we find that our new nonbonded potentials provide good agreement with QM and experimental data.
\end{abstract}

Published under license by AIP Publishing. https://doi.org/10.1063/1.5113811

\section{INTRODUCTION}

Molecular dynamics (MD) simulations have become an essential partner with experiment, playing a major role in elucidating physical and chemical properties of systems and in developing new generations of materials. Ideally, one would use quantum mechanical (QM) methods to make the most accurate predictions of the properties of new systems. However, QM methods are computationally limited to hundreds of atoms for dozens of picoseconds, which is inadequate for most developments of new generations of catalysts, materials, and pharmaceutical molecules, particularly for electrochemical and biological systems where an explicit description of the solvent is important. A system just $25 \mathrm{~nm}$ on a side already requires millions of atoms for time scales of microseconds or more, far beyond the realm of QM. Thus, we need to develop force fields (FFs) that retain the accuracy of QM while scaling to millions of atoms and microseconds.

Most FFs distinguish between short range valence interactions and long-range nonbonded interactions. The valence interactions may be cast in terms of bond distances, angles, torsion, and inversion terms. The long-range nonbonded interactions include electrostatics, polarization, and van der Waals (vdW) terms (which account for London dispersion and Pauli repulsion). In addition, hydrogen bond (HB) corrections are often needed 
to describe short-range corrections to the nonbonded interactions.

Many FFs have been developed for simulations of various systems including biological systems (OPLS, AMBER, ${ }^{2}$ and $\mathrm{CHARMM}^{3}$ ), water (TIP4P-2005, ${ }^{4} \mathrm{~mW},{ }^{5}$ SPC/Ew, ${ }^{6}$ and TIP3P ${ }^{7}$ ), membranes, ${ }^{8,9}$ energetic materials, ${ }^{10}$ and catalytic reactions. ${ }^{11,12}$ Some of these FFs are generic (e.g., $\mathrm{UFF}^{13}$ ), and some can describe bond breaking and reactions (e.g., ReaxFF ${ }^{14}$ ).

FFs use a variety of functional forms for the nonbonded potentials, including the Lennard Jones (LJ) 12-6 (AMBER, OPLS, ${ }^{1}$ DREIDING, ${ }^{15}$ CHARMM $22,{ }^{3}$ and GROMOS ${ }^{16}$ ), Buckingham exponential-6 and its modified version (MM2, ${ }^{17}$ MM3, DREIDING X6, ${ }^{15}$ APPLE\& $\mathrm{P}^{19}$ ), and buffered 14-7 (MMFF, AMOEBA $^{21}$ ).

The quality of these FFs depends mainly on the accuracy of the parameters, particularly beyond the training set.

Mainly two approaches have been used to determine the parameters of the nonbonded potentials. In the first approach, the parameters of the potentials are fitted to reproduce one or more experimental properties. For example, empirical water FFs (e.g., TIP4P-2005, and SPC/Ew) were developed by fitting the parameters of the models to reproduce two or more experimental properties such as density, heat of vaporization, diffusion, and/or melting point. Biological FFs such as OPLS, AMBER, and CHARMM FFs often focused on properties of proteins or nucleic acids. A major problem of such FFs is that their parameters are based on reproducing experimental quantities for specific systems, limiting their accuracy for novel systems. See Refs. 22 and 23 for a comparison between the accuracy of different water models that are fitted to reproduce different experimental properties.

The second approach uses QM based data to optimize parameters. This may provide potentials with more accurate descriptions of chemical and physical interactions at the atomistic level. Also, it makes the FF more suitable for novel systems under conditions not accessible to experiment. However, the quality of QM based FFs depends highly on the level of QM and how these data are used to optimize the FF parameters.

Usually, the QM data for relatively small molecular structures are used for parameter fitting. For example, the MB-pol ${ }^{24}$ and CC$\mathrm{pol}^{25}$ ab initio based water models were developed by fitting potentials and many-body terms to a large data set of water dimers and trimers using high quality QM data.

The QM data for such small nonperiodic molecular structures may not sample the long-range interactions important in multiscale simulations of bulk materials.

Sometimes, the FF parameters are extracted from QM calculations on crystals (periodic systems) by fitting to quantities such as bulk moduli, elastic constant, and lattice parameters. Then, the parameters of the nonbonded potentials are optimized together to agree with QM. Here, the problem is for systems containing several atomic types, where there is not a clear methodology to separate the contributions of $\mathrm{vdW}$, electrostatics, and polarization terms from the QM. Thus, such FFs may perform well only for the particular system that was fitted to and are often not transferable to new systems.

Some FFs (e.g., AMBER and OPLS) use QM partial atomic charges as the reference data points to establish the electrostatics energy term separately. ${ }^{26}$ The problem here is that the electron population analysis (EPA) to convert the QM electron density to partial atomic charges is ambiguous. Many EPA methods are used, including Mulliken population analysis (MPA), ${ }^{27,28}$ Löwdin population analysis (LPA), ${ }^{29,30}$ and electrostatic potential (ESP). ${ }^{27}$ These methods sometimes result in very different atomic charges for similar molecular structures (see Ref. 31 for more details). Therefore, the value and quality of the electrostatic energy term are significantly affected by quality of QM based partial atomic charges. Some FFs (e.g., CHARMM) use the $a b$ initio dipole moments of molecules to obtain the fixed atomic charges. Here, the use of fixed charges avoids the need for molecular charge distribution to be updated based on the changes in the electrostatic environment.

Our goal is to develop a new strategy that allows us to separately define different components of the nonbonded potential based on accurate periodic QM (energy or forces) calculations.

For the electrostatic interactions, we use our recent polarizable charge equilibration (PQEq) model. ${ }^{8}$ PQEq uses an atomic sized Gaussian shaped electron density (not point charges) that can polarize away from the core (using a Gaussian function to describe the shell) in response to internal and external electric fields. The atomic charges are updated dynamically to achieve a constant chemical potential across all atoms in the system. An important feature of PQEq is that all atomic parameters are validated by comparing to QM interaction energy (not the partial charges) of electric probe dipoles. ${ }^{31-33}$ Here, the interaction energy includes only electrostatics and polarization effects, which helps to optimize directly the parameters of PQEq based on QM data.

Next, we need to develop a methodology to describe the vdW interactions based on accurate density functional theory (DFT) methods. We found that DFT including the Grimme ${ }^{34,35}$ vdW or London dispersion corrections (D2 and D3) leads to energy-volume equations of state (EOSs) for molecular solids in good agreement with experiment. ${ }^{23}$ Thus, we report here the two-body nonbonded interaction parameters based on fitting to the QM EOS for several main group single element crystals including $\mathrm{H}, \mathrm{C}, \mathrm{N}, \mathrm{O}, \mathrm{F}, \mathrm{Cl}, \mathrm{Br}$, I, $\mathrm{P}, \mathrm{He}, \mathrm{Ne} \mathrm{Ar}, \mathrm{Kr}, \mathrm{Xe}$, and $\mathrm{Rn}$ elements. We consider that these parameters capture the pure vdW interactions since the electrostatic and polarization contributions are negligible for these molecular systems (see Sec. V).

Together, the vdW and PQEq interactions provide the long range or nonbonded part of our new generation reactive $\mathrm{FF}^{23}$ (RexPoN). RexPoN uses QM optimized polarization (Po), QM based nonbonded $(\mathrm{N})$, and QM based bond dissociation (Rex). For systems such as water and biological systems, we allow the explicit hydrogen bond (HB) term to tune the short range nonbonded interaction (see Sec. VI). This paper does not discuss PQEq and the valence interactions, the details of which are provided in our previous publications. ${ }^{23,31-33}$

The present study focuses on the development of the vdW nonbonded potentials using the EOS of the single element crystals. For simplicity, we refer to this as the "nonbonded" interactions.

Thus, we determined the individual two-body nonbonded potentials for each of the 15 above elements by fitting to the energyvolume EOS data (called $E_{P R-L D}$ ). These fittings make no assumptions about the functional form (e.g., LJ, Morse, or Buckingham). Rather, we use a general Rydberg expansion to provide the best fit to the reference data. 
We discovered that scaling the nonbonded potential curves of the noble gases by normalizing the well depth energy $\left(D_{e}\right)$, bond distance $\left(R_{e}\right)$, and curvature using a scaling length $(L)$ led to nearly identical scaled potentials. Having such a universal nonbonded potential requiring only three parameters for each element would be most valuable for extending nonbonded interactions to the rest of the periodic table for elements that do not lead to molecular crystals. Thus, we define a universal nonbonded potential $\left(E_{U N B}\right)$ by averaging the individual scaled potential for each of the 6 noble gas atoms. For all 15 elements, we have extracted the $D_{e}, R_{e}$, and $L$ that fit $E_{U N B}$.

This paper is organized as follows. Section II describes the crystal structures and energy-volume EOS calculations for 15 main group elements. Section III provides the details of fitting EOS to the nonbonded potentials. Section IV details the derivation of the universal nonbonded potential, which can be the starting point for the remaining elements of the periodic table. Section $V$ discusses our methodology. Section VI reports the application of RexPoN to several systems. The conclusions are given in Sec. VII.

\section{CRYSTAL STRUCTURES AND ENERGY-VOLUME EQUATIONS OF STATE (EOSs)}

We used DFT-D3 QM to compute the energy-volume EOS of some of the main group elements including $\mathrm{H}, \mathrm{C}, \mathrm{N}, \mathrm{O}, \mathrm{P}$, halogens ( F, Cl, Br, and I), and noble gases (Ne, He, Ar, Kr, Xe, and Rn). For all cases, we started from the most stable crystal structures obtained from the literature. Then, we changed the volume of the cells to calculate the single point energies vs volume. For cases with bonds, we fixed the bond distances and angles to avoid contributions from valence terms. We compressed the cells to small volumes to obtain the short-range nonbonded interactions corresponding to high pressures (above $100 \mathrm{GPa}$ ). Here, we provide a short description of each system.

Hydrogen: X-ray diffraction shows that the $\mathrm{H}_{2}$ crystal has a hexagonal closed packed structure with the $P 6_{3} / m m c$ space group and $c / a$ ratio close to the ideal value of $1.633 .{ }^{36}$ However, since hydrogen has no core electrons, X-ray experiments cannot determine the exact position of the $\mathrm{H}$ atom coordinates. Therefore, extensive theoretical studies have been carried out to determine the $\mathrm{H}_{2}$ molecule orientation. It was found that the $\mathrm{Pca} 2_{1}$ space group (see Fig. 1) containing a hexagonal close packed structure with four molecules per unit cell is the most stable structure at high pressure ranges $(\sim 110-150 \mathrm{GPa}){ }^{37,38}$ The equilibrium bond distance of $\mathrm{H}-\mathrm{H}$ is $0.74 \AA$.

Oxygen: For solid oxygen, we selected the stable $\alpha$-phase crystal structure, which according to neutron-diffraction and X-ray measurements has a monoclinic base-center structure with $C 2 / m$ space group $^{39,40}$ and O-O bond distance of $1.207 \AA$.

Carbon: To compute the EOS of carbon, we used the alpha hexagonal structure of graphite with the $\mathrm{PG}_{3} / \mathrm{mmc}$ space group (ABAB stacking sequence). ${ }^{41}$ The hexagonal rings in the plane are covalently bonded with the $\mathrm{C}-\mathrm{C}$ bond lengths of $1.424 \AA$.
A) Hydrogen

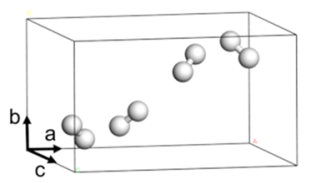

D) Phosphorus

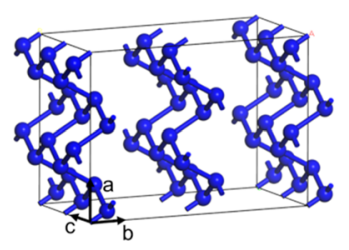

G) Chlorine

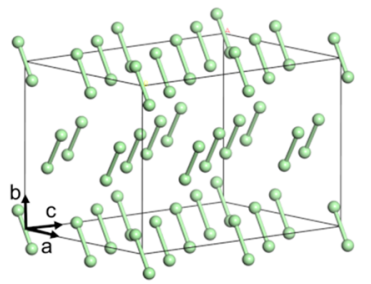

B) Graphite

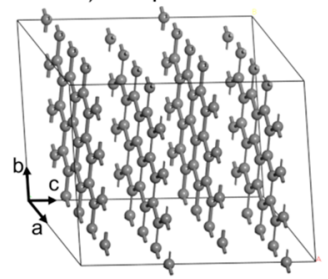

E) Oxygen

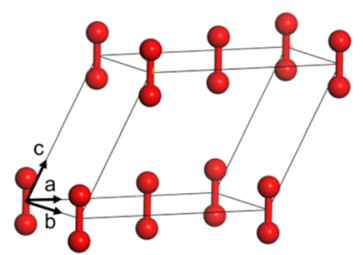

H) Bromine

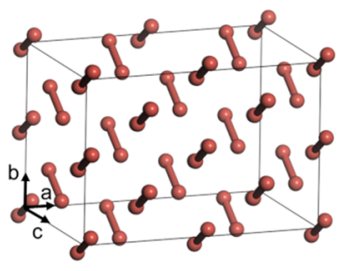

C) Nitrogen (alpha)

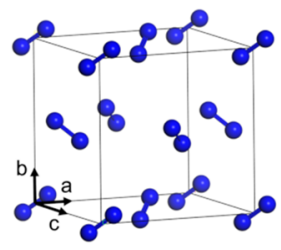

F) Fluorine

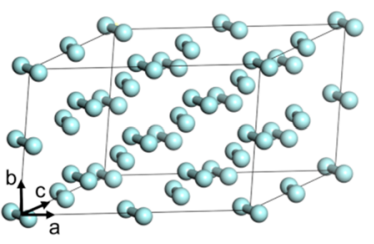

I) lodine

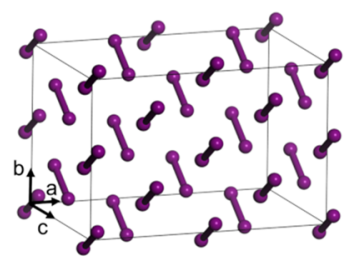

FIG. 1. Crystal structures of (a) hydrogen, (b) graphite, (c) nitrogen (alpha phase), (d) black phosphorous (BP) (also used for nitrogen BP), (e) oxygen, (f) fluorine, $(\mathrm{g})$ chlorine, (h) bromine, and (i) iodine. Supercells are included in (b), (d), (f), (g), (h), and (i) in order to show the molecular structures better. 
Nitrogen: We selected two systems for nitrogen crystals. The first structure is the alpha- $\mathrm{N}_{2}$ structure that exists below $35.6 \mathrm{~K}$ with the $\mathrm{Pa} 3$ space group. ${ }^{42}$ The bond distance of $\mathrm{N}_{2}$ molecules is $1.112 \AA$. The second structure is the black phosphorous structure (see below) where we replaced the phosphorus atoms with nitrogen. The reason for including this structure is to examine the nonbonded interactions between nitrogen atoms that have single bonds rather than a triple bond.

Phosphorus: The stable black-phosphorous (BP) structure is orthorhombic with Cmca space group. The BP structure is stable at ambient conditions. ${ }^{43}$ The crystal structure consists of interconnected six-membered rings with 2.256 and $2.227 \AA$ A bond distances.

Halogens: For fluorine, we used the crystal structure of $\alpha-F$ determined by $\mathrm{X}$-ray powder diffraction at $23 \mathrm{~K}$ with $C 2 / c$ space group $^{44}$ and the F-F bond distance of $1.45 \AA$. For chlorine, bromine, and iodine, we used the crystal structures determined by neutron diffraction at low temperatures which have $\mathrm{Cmca}$ space groups. We used solid crystals of $\mathrm{Cl}_{2}$ at $22 \mathrm{~K}^{45}$ for $\mathrm{Cl}, \mathrm{Br}_{2}$ at $5 \mathrm{~K}^{45}$ for $\mathrm{Br}$, and $\mathrm{I}_{2}$ at $15 \mathrm{~K}^{46}$ for $\mathrm{I}$. The bond distances in the cells are $2.01 \AA$ for $\mathrm{Cl}_{2}, 2.30$ $\AA$ for $\mathrm{Br}_{2}$, and $2.72 \AA$ for $\mathrm{I}_{2}$.

Noble gases: All the noble gas crystal structures were produced by scaling the cell size of a face-centered cubic structure, which is known to be the most stable form for noble gases crystals. ${ }^{4}$

The crystal structures of $\mathrm{H}, \mathrm{C}, \mathrm{N}, \mathrm{O}, \mathrm{P}, \mathrm{F}, \mathrm{Cl}, \mathrm{Br}$, and I systems are shown in Fig. 1.

The DFT-D3 energy-volume EOSs are shown in Fig. 2 for $\mathrm{H}$, C, N, O, and P elements, in Fig. 3 for halogens, and in Fig. 4 for noble gases (black open circles). The solid red lines show the fitting of RexPoN nonbonded potentials to the DFT-D3 data, as described in Sec. III.

To avoid any contribution from changes in the covalent bonds, we kept all bond lengths and angles fixed at the value for the stable/equilibrium structure. To compute the energy for each volume, we subtract the total energy of the cell from the reference molecule with the same bond lengths and angles in all cells. See the supplementary material for more details.

We calculated the EOS for all systems using the Perdew-BurkeErnzerhof (PBE) $)^{32}$ flavor of DFT. The only exception is for solid oxygen crystal, for which we used Becke, three-parameter, LeeYang-Parr (B3LYP) ${ }^{48,49}$ including the hybrid terms important for describing the $\mathrm{O}_{2}{ }^{3} \Sigma_{\mathrm{g}}{ }^{-}$triplet state, ${ }^{35,36}$ which is poorly described by PBE. See more discussion about the EOS of $\mathrm{O}_{2}$ in Ref. 23. To account for long-range vdW interactions, we used DFT-D3 Grimme dispersion correction with the Becke-Johnson parameters. ${ }^{35,50}$ See the supplementary material for more information about DFT-D calculations.

The cell volumes were scaled isotropically for all noble gases, $\mathrm{H}, \mathrm{N}$ (alpha), and $\mathrm{O}$ crystals. For $\mathrm{C}, \mathrm{F}, \mathrm{Cl}, \mathrm{Br}$, and I, we changed the cells along the direction perpendicular to the molecular planes, while for $\mathrm{P}$ and $\mathrm{N}$ (BP), we changed the cells along $b$ direction. The valence bonds and angles were kept fixed during the EOS calculations.

We compressed the volumes of the cells to achieve pressures up to $120 \mathrm{GPa}$ for $\mathrm{H}, 315 \mathrm{GPa}$ for C, $332 \mathrm{GPa}$ for $\mathrm{N}$
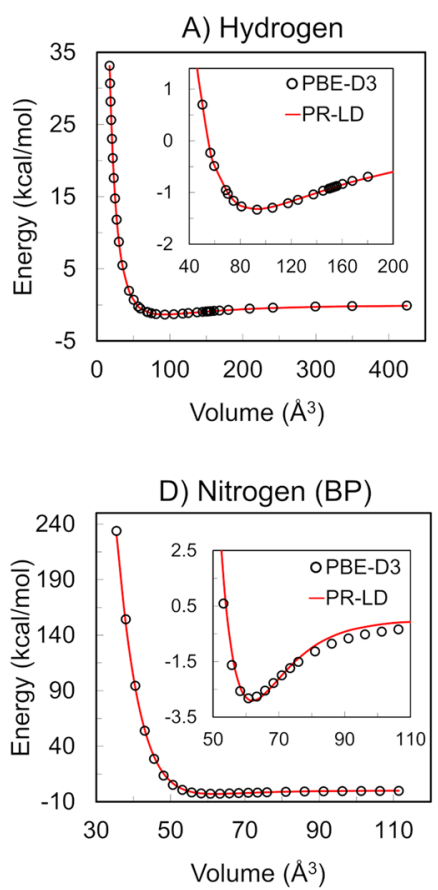
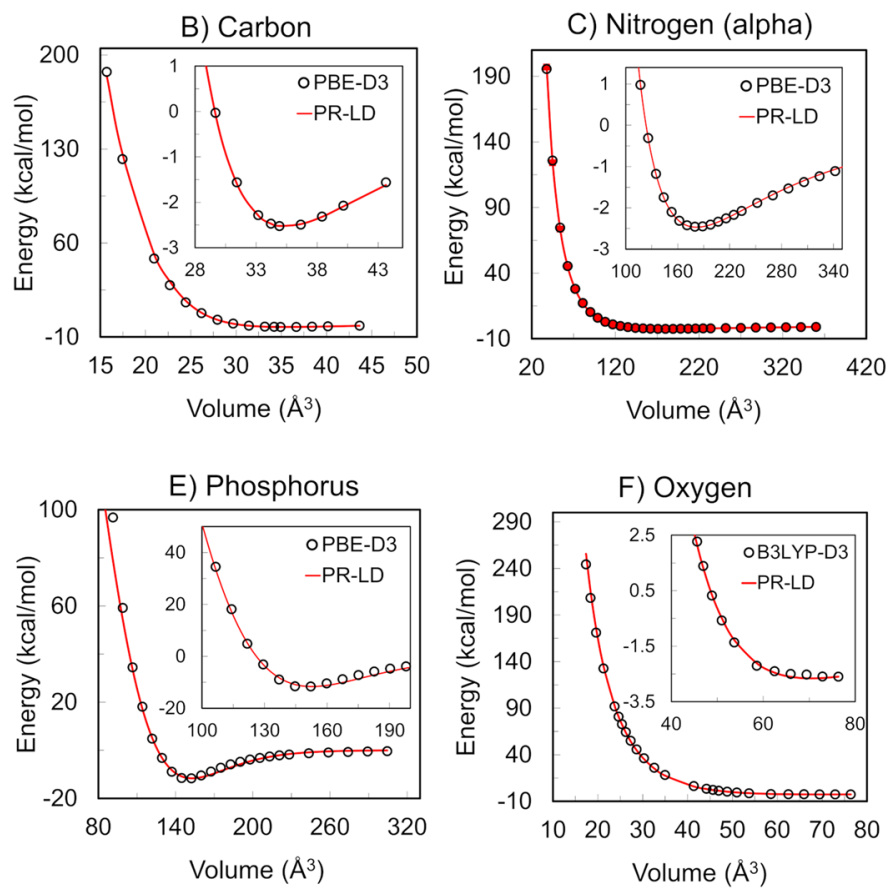

FIG. 2. The vdW energy-volume EOS computed by DFT-D3 and RexPoN for solid (a) hydrogen, (b) graphite, (c) nitrogen (alpha phase), (d) nitrogen (black-phosphorus structure), (e) black phosphorus, and (f) oxygen. The two-body $E_{P R-L D}$ nonbonded potential function given in Eq. (1) (red line) was fitted to each DFT-D to obtain the adjustable parameters. These parameters are listed in Table I. 
A) Fluorine

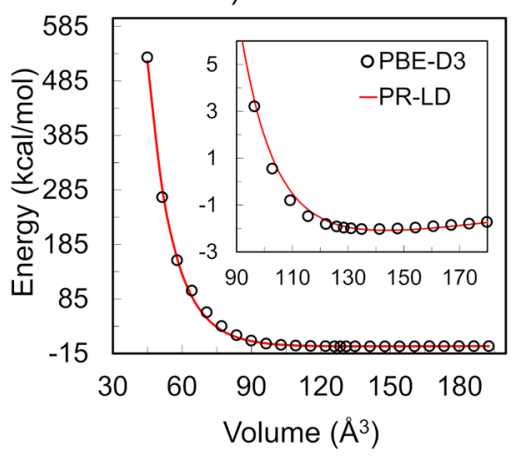

C) Bromine

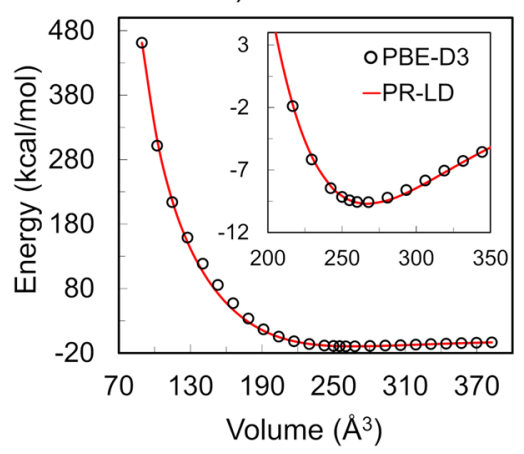

B) Chlorine

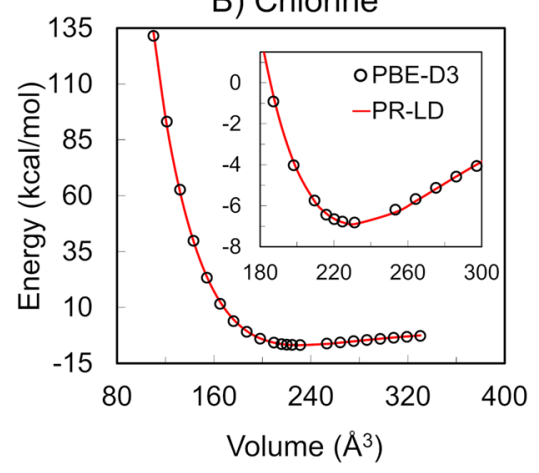

D) lodine

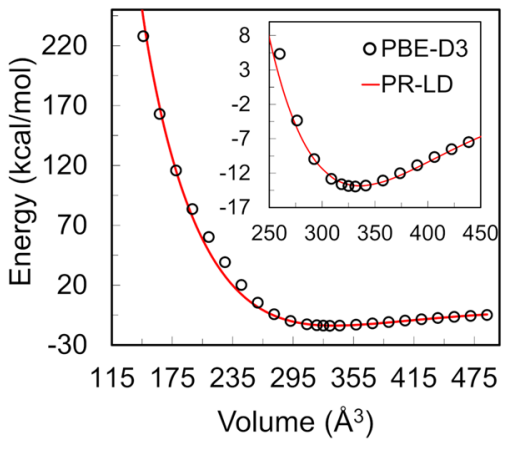

FIG. 3. Same as Fig. 2 but for halogen atoms including solid (a) fluorine, (b) chlorine, (c) bromine, and (d) iodine.

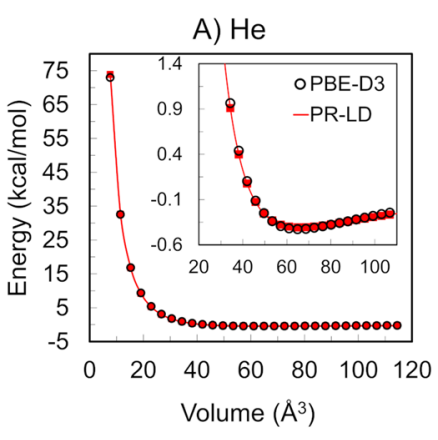

D) $\mathrm{Kr}$



B) $\mathrm{Ne}$

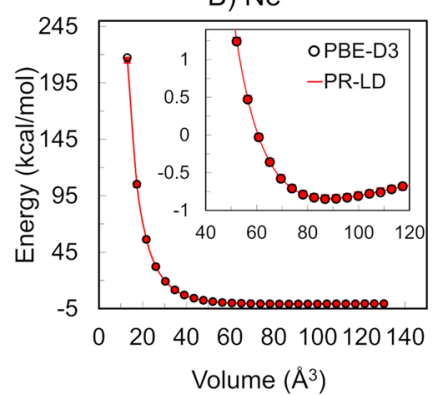

E) $\mathrm{Xe}$

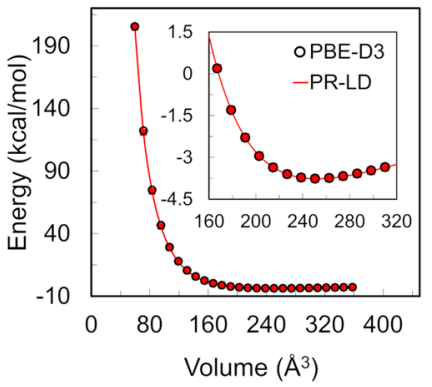

C) $\mathrm{Ar}$

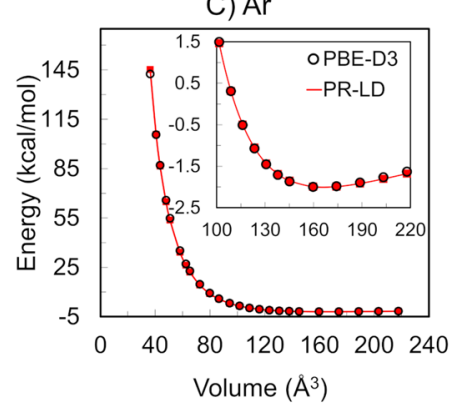

F) $\mathrm{Rn}$

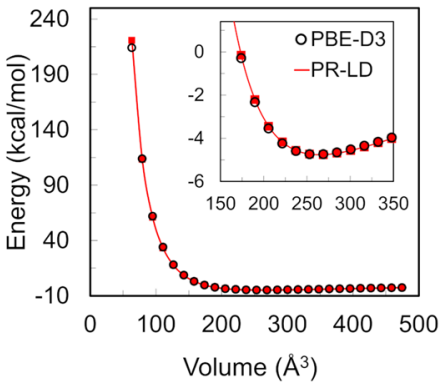

FIG. 4. Same as Fig. 2 but for noble gases including (a) $\mathrm{He}$, (b) $\mathrm{Ne}$, (c) $\mathrm{Ar}$, (d) $\mathrm{Kr}$, (e) $\mathrm{Xe}$, and (f) $\mathrm{Rn}$. 
(440 GPa for $\mathrm{N}$ in the $\mathrm{BP}$ structure), $300 \mathrm{GPa}$ for $\mathrm{O}, 194 \mathrm{GPa}$ for $\mathrm{P}$, up to $440 \mathrm{GPa}$ for halogens, and up to $2000 \mathrm{GPa}$ for noble gases.

\section{FITTING THE NONBONDED POTENTIAL}

We fitted two body vdW nonbonded interaction functions to the computed energy-volume EOS from DFT-D. Our nonbonded potential is defined to capture general repulsive interaction arising from the Pauli principle (also referred to a vdW repulsion), denoted here as PR, and the attractive interactions arising from London dispersion (or vdW attraction), denoted here as LD. For PR, we use a combination of two exponential terms, and for London dispersion, we use a low-gradient (LG) functional form as was proposed by Liu and Goddard ${ }^{51}$ and used in the LD corrections to DFT by Kim et al. ${ }^{52}$ The sum of the PR and LD terms forms our nonbonded potential (PR-LD),

$$
\begin{aligned}
E_{P R-L D}=E_{P R}+E_{L D}= & \sum_{i<j} A_{i j} \exp \left(s_{i j} r_{i j}+\beta_{i j}\right) \exp \left(\gamma_{i j} r_{i j}^{n_{i j}}+\eta_{i j} r_{i j}+\delta_{i j}\right) \\
& \times \operatorname{Tap}\left(r_{i j}\right)-\sum_{i<j} \frac{C 6_{i j}}{r_{i j}^{6}+\left(2 R_{v d W i j}\right)^{6}} \operatorname{Tap}\left(r_{i j}\right), \quad(1)
\end{aligned}
$$

where $r_{i j}$ is the interatomic distance. The first sum is designed to be a monotonic purely repulsive (positive) term with $A_{i j}, s_{i j}, \beta_{i j}$, $\gamma_{i j}, n_{i j}, \eta_{i j}$, and $\delta_{i j}$ being adjustable parameters. The second sum is a monotonic attractive term with $C 6_{i j}$ being the dispersion energy parameter and $R_{v d W_{i j}}$ being the equilibrium vdW distance between atoms $i$ and $j$. This term goes as $1 / r_{i j}{ }^{6}$ for long $r_{i j}$ but has an inflection point at $R_{v d W i j}$ becoming constant and small for normal bond distances. All of the parameters in Eq. (1) depend only on the atomic types.

The $\operatorname{Tap}\left(r_{i j}\right)$ is a 7 th order taper function with a finite range which is used to screen the nonbonded interactions. The taper function makes the nonbonded potential energy and its first three derivatives go smoothly to zero at a finite distance,

$$
\operatorname{Taper}\left(r_{i j}\right)=\sum_{m=0}^{7} \operatorname{Tap}_{m}\left(\frac{r_{i j}}{r_{c u t}}\right)^{m} \text {. }
$$

Here, $r_{c u t}$ is a cutoff length $(12.0 \AA)$ and $\mathrm{Tap}_{7}=20$, $\mathrm{Tap}_{6}=-70$, Tap 5 $=84, \operatorname{Tap}_{4}=-35, \operatorname{Tap}_{3}=0, \operatorname{Tap}_{2}=0, \operatorname{Tap}_{1}=0$, and Tap 01 .

We used a step-by-step optimization strategy to obtain the parameters in Eq. (1). We performed the parameter optimization for each atomic type separately. We constructed the training set by subtracting the total DFT-D energy of the crystals (for every volume along the EOS curve) from the energy of the reference structure. See Sec. II of the supplementary material for more details about reference structures. Throughout the optimization, we minimized the error (cost) function given by

$$
\text { Error }=\sum_{i=0}^{N_{t}} \frac{1}{w_{i}^{2}}\left(E_{D F T-D}-E_{P R-L D}\right)^{2},
$$

where $N_{t}$ is the number of items in the training set and $w_{i}$ is the weight for each data point in the training set.

We started with the $E_{L D}$ term [second sum in Eq. (1)] and used the initial values for $R_{v d W i j}$ and $C 6_{i j}$ from the Universal Force Field (UFF) (available up to lawrencium, $Z=103$ ). ${ }^{13}$ Our initial goal is to modify these two parameters in such a way that the value of the $E_{L D}$ term becomes twice as deep as the DFT-D value at the volume corresponding to the well depth $\left(V_{w}\right)$ of EOS (i.e., $E_{L D}=-2 E_{w}$ at $\left.V_{w}\right)$. The DFT-D value for the total binding leads to $-E_{w}$, which is the sum of a positive PR term and the London dispersion. We assume that at $V_{w}, E_{L D}$ is $-2 E_{w}$ and $E_{P R}$ is $+E_{w}$.

Since the UFF values for $R_{v d W i j}$ are reasonably accurate, we modified only the $C 6_{i j}$ parameter (defined based on atomic polarizabilities in UFF) to satisfy this condition. However, we optimize

\begin{tabular}{|c|c|c|c|c|c|c|c|c|c|}
\hline Atom & $A$ & $s$ & $\beta$ & $\gamma$ & $n$ & $\eta$ & $\delta$ & $R v d W$ & C6 \\
\hline $\mathrm{H}$ & 0.0212 & -2.4002 & 10.5460 & -0.8113 & 0.0014 & -0.2607 & -0.5640 & 1.6346 & 239.4803 \\
\hline $\mathrm{He}$ & 0.0143 & -3.5531 & 11.7112 & 0.0000 & 1.0000 & 0.0000 & 0.0000 & 1.1937 & 87.9969 \\
\hline $\mathrm{C}$ & 0.2537 & -2.4545 & 1.8207 & 0.3108 & 0.8248 & -0.4569 & 7.7134 & 1.3400 & 792.9588 \\
\hline $\mathrm{N}$ (alpha) & 0.3670 & -3.7952 & 5.5977 & 4.4641 & 0.4424 & 0.0010 & 0.0010 & 1.1781 & 1000.9191 \\
\hline $\mathrm{N}(\mathrm{BP})$ & 8.4296 & -2.9375 & 6.8368 & -0.0004 & 0.6089 & 0.0010 & -0.0008 & 1.0564 & 593.9540 \\
\hline $\mathrm{O}$ & 0.0272 & -3.6468 & 14.5278 & -0.3235 & 0.0305 & 0.0331 & -0.5688 & 1.4969 & 634.7066 \\
\hline $\mathrm{F}$ & 0.7710 & -3.9612 & 10.0284 & 0.0286 & 2.8974 & 0.1941 & -0.0086 & 1.6762 & 921.9329 \\
\hline $\mathrm{Ne}$ & 0.1048 & -4.0308 & 12.4882 & 0.0029 & 0.9966 & 0.0163 & 0.0423 & 1.3518 & 291.8884 \\
\hline $\mathrm{P}$ & 3.6525 & -2.0073 & 6.7334 & -0.4335 & 0.5155 & 0.5514 & -1.3245 & 2.0000 & 13000.0000 \\
\hline $\mathrm{Cl}$ & 3.4737 & -2.6483 & 8.4472 & 0.1872 & 0.6288 & 0.0124 & -0.3986 & 1.4241 & 3680.2392 \\
\hline Ar & 2.2852 & -3.1609 & 9.8446 & 0.0027 & 0.9965 & -0.0136 & 0.0482 & 1.9108 & 3425.3776 \\
\hline $\mathrm{Br}$ & 5.7482 & -1.5770 & 4.7932 & -0.0783 & 1.2980 & -0.4349 & 2.1154 & 1.6561 & 8502.2025 \\
\hline $\mathrm{Kr}$ & 10.4740 & -2.8331 & 8.2831 & 0.0002 & 1.0000 & 0.0001 & -0.0001 & 1.9195 & 6579.6328 \\
\hline I & 1.7070 & -2.3274 & 9.1643 & -0.5343 & 0.5218 & 0.1545 & 0.4811 & 2.2828 & 22100.0000 \\
\hline $\mathrm{Xe}$ & 14.2394 & -2.6297 & 8.2880 & 1.4575 & 0.7617 & -0.9165 & -0.6258 & 2.2992 & 18800.6328 \\
\hline $\mathrm{Rn}$ & 16.0833 & -2.6363 & 8.4742 & 0.0000 & 1.0000 & 0.0000 & 0.0000 & 2.4949 & 33000.0000 \\
\hline
\end{tabular}
parameters in later steps, so the final values for $R_{v d W i j}$ and $C 6_{i j}$ may differ from the starting parameters.

TABLE I. Parameters of nonbonded potential energy term $\left(E_{P R-L D}\right)$ given in Eq. (1). 
Next, we performed the optimization of $A_{i j}, s_{i j}$, and $\beta_{i j}$ in the first exponential term of $E_{P R}$. Our goal here is to minimize the cost function in Eq. (3) to get the best fit. Once reasonable agreement is obtained to the reference values, we turn on optimization of the parameters in the second exponential term $\left(\gamma_{i j}\right.$, $n_{i j}, \eta_{i j}$, and $\left.\delta_{i j}\right)$ to improve the quality of the fit. Here, the quality of the $E_{P R}$ energy term is mainly controlled by the first exponential term. In some cases, we obtained the final parameters just by optimizing the $E_{L D}$ term and the first exponential term of $E_{P R}$. Finally, we performed a global optimization (when needed) of all parameters together to further minimize the value of the cost function.

There are two important reasons for using the above step-bystep optimization strategy. First, it helps to avoid overfitting of the parameters. Thus, the parameters obtained are not biased toward the training set and have better global character. Second, it helps to define more physical repulsive and attractive energy curves rather than just trying to reduce the value of cost function.

Initially, we used $w_{i}=1$ for all data points in the training set. However, during the global optimization step, we assigned smaller $w_{i}$ values to the training data points around the well depth to increase the accuracy of the fit around the equilibrium. Here, we varied the value of $w_{i}$ from 0.1 to 1.0. More details about the optimizer engine are provided in the supplementary material.

The fits for the above elements are shown in Figs. 2-4 (solid red lines). We find an excellent fit for all cases. Therefore, $E_{P R-L D}$ of RexPoN provides a description of the short- and long-range nonbonded interaction for each element at the accuracy level of DFT-D3. The optimized parameters of $E_{P R-L D}$ are provided in Table I.

\section{UNIVERSAL NONBONDED POTENTIAL FUNCTION}

The EOS of noble gases provides pure nonbonded interactions since there are no valence or electrostatic interactions.

In order to analyze the functional form for these nonbonded vdW interactions, we use the same scaling methodology proposed by Rose et al. ${ }^{53}$ We scale the curves such that at the minimum position of the potential well $\left(R_{e}\right)$, the dimensionless values of energy and second derivative (i.e., curvature) are both equal to one. Therefore,
TABLE II. The $R_{e}, D_{e}$, and $L$ atomic parameters obtained from two-body $E_{P R-L D}$ potential energy curves [Eq. (1)].

\begin{tabular}{lccc}
\hline \hline Atoms & $R_{e}(\AA)$ & $D_{e}(\mathrm{kcal} / \mathrm{mol})$ & $L(\AA)$ \\
\hline $\mathrm{H}$ & 3.2541 & 0.0528 & 0.5241 \\
$\mathrm{He}$ & 2.9752 & 0.0553 & 0.5241 \\
$\mathrm{C}$ & 3.9162 & 0.0971 & 0.5396 \\
$\mathrm{~N}$ (alpha) & 3.8281 & 0.1456 & 0.5416 \\
$\mathrm{~N}(\mathrm{BP})$ & 3.8918 & 0.0814 & 0.5170 \\
$\mathrm{O}$ & 3.4249 & 0.1498 & 0.4349 \\
$\mathrm{~F}$ & 3.5018 & 0.1873 & 0.5199 \\
$\mathrm{Ne}$ & 3.2434 & 0.1229 & 0.4121 \\
$\mathrm{P}$ & 4.2238 & 0.6297 & 0.7194 \\
$\mathrm{Cl}$ & 4.0748 & 0.3500 & 0.5654 \\
$\mathrm{Br}$ & 4.3103 & 0.5143 & 0.6318 \\
$\mathrm{Ar}$ & 4.0336 & 0.3359 & 0.5604 \\
$\mathrm{Kr}$ & 4.2715 & 0.4790 & 0.5825 \\
$\mathrm{I}$ & 4.6316 & 0.8256 & 0.7161 \\
$\mathrm{Xe}$ & 4.6476 & 0.7316 & 0.6748 \\
$\mathrm{Rn}$ & 4.7938 & 0.9468 & 0.7464 \\
\hline \hline
\end{tabular}

we use the well depth energy $\left(D_{e}\right), R_{e}$, and a scaling length parameter $(L)$ and set

$$
\begin{aligned}
& \rho=\frac{R-R_{e}}{L}, \\
& \varepsilon=\frac{E}{D_{e}},
\end{aligned}
$$

and

$$
\frac{\omega L^{2}}{D_{e}}=1
$$

where $\omega$ is the curvature or the value of the second derivative of the potential curve at $R_{e}$ (at $r=R_{e}, \omega=d^{2} E / d r^{2}$ ). Equation (6) is used to determine the value of $L$ which makes the dimensionless curvature at $\rho$ equal to 1 . We use the $E_{P R-L D}$ two-body potential energy curves to obtain the atomic parameters $\left(R_{e}, D_{e}\right.$, and $\left.L\right)$ for
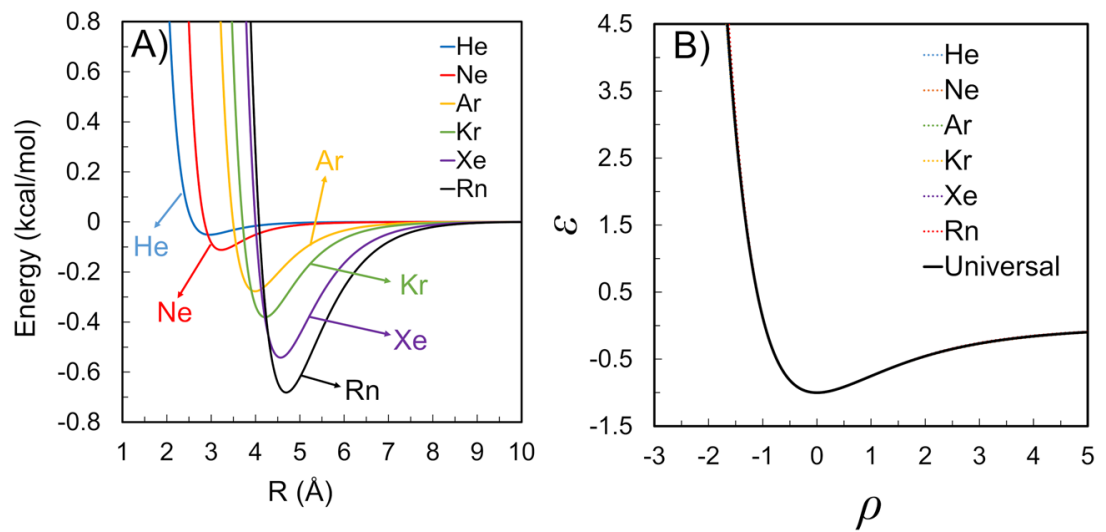

FIG. 5. (a) The two-body nonbonded or vdW potential energy curves of noble gas elements. (b) The scaled energy curves obtained using the minimum well depth distance $\left(R_{e}\right)$, energy $\left(D_{e}\right)$, and the scaling length $(L)$ using Eqs. (4)-(6). 
TABLE III. Parameters of the universal nonbonded energy curve $\left(E_{U N B}\right)$ given in Eq. (8).

\begin{tabular}{lcccccc}
\hline \hline$\beta$ & $\alpha_{0}$ & $\alpha_{1}$ & $\alpha_{2}$ & $\alpha_{3}$ & $\alpha_{4}$ & $\alpha_{5}$ \\
\hline 1.0035 & 1.0000 & 1.0201 & 0.0168 & 0.0033 & 0.0037 & 0.0011 \\
\hline \hline
\end{tabular}

each element. The atomic parameters are listed in Table II. Using Eqs. (4)-(6), the scaled two-body potential energy curves are shown in Fig. 5(b). We note that the scaled curves are nearly identical (with a root mean square deviation in $\varepsilon$ of 0.02 over the range $\rho=-1.5-5$ ), suggesting a universal nonbonded vdW potential that can be used for all elements of the periodic table. Using this universal curve, the two-body vdW interaction energy is defined by just the three parameters $\left(R_{e}, D_{e}\right.$, and $\left.L\right)$ for each element. For interactions involving two or more elements, we use the geometric mean combination rules.

We averaged all the scaled curves in Fig. 5(b) to develop the universal scaled nonbonded curve which was then fitted to an extended Rydberg-type equation ${ }^{54}$ given by

$$
\varepsilon_{U N B}\left(\rho_{i j}\right)=-\exp \left(-\beta \rho_{i j}\right) \sum_{n=0}^{5} \alpha_{n} \rho_{i j}^{n},
$$

where $\beta$ and $\alpha_{n}$ are the universal parameters with the values provided in Table III.

The scaled potential in Eq. (7) can be converted back to the real space using the relations given in Eqs. (4)-(6) leading to the universal nonbonded potential $\left(E_{U N B}\right)$ function given by

$$
E_{U N B}\left(r_{i j}\right)=-D_{e} \exp \left[-\beta\left(\frac{r_{i j}-R_{e}}{L}\right)\right] \operatorname{Tap}\left(r_{i j}\right) \sum_{n=0}^{5} s_{n}\left(\frac{r_{i j}-R_{e}}{L}\right)^{n} .
$$

We add a similar 7 th order taper function as given in Eq. (2) to screen the nonbonded interactions. Therefore, the nonbonded potential for any element can be obtained at the level of DFT-D with just the three parameters of the two-body nonbonded potentials.

This universal nonbonded curve should simplify estimating vdW nonbonded parameters for the elements of the periodic table which do not form molecular crystals dominated by vdW interactions (e.g., transition metals). Here, we hope to extrapolate and interpolate the $D_{e}, R_{e}$, and $L$ parameters to estimate unknown cases. Our goal is to provide an accurate universal potential (at the accuracy level of DFT-D) to describe the interatomic interactions for systems near equilibrium. As such, we chose the distances around the vdW potential well depth $(\rho=-1.5-5)$. This helps avoid differences between nonbonded potential curves at very close distances on $E_{U N B}$.

We have integrated both the $E_{P R-L D}$ and $E_{U N B}$ functional forms into our local LAMMPS program software.

\section{DISCUSSION}

\section{A. Contribution of polarization energy to EOS}

Our strategy is to use the computed EOS curve for single element crystals to define the nonbonded parameters in which electrostatic and polarization terms (described by PQEq) vanish. The PQEq model allows the charge distribution to change according to the electrostatic environment. The optimum charge distribution $\left(q_{i}\right)$ and consequently the shell polarizations are computed from the conditions that the chemical potentials $\left(\partial E_{P Q E q} / \partial q_{i}\right)$ be equal for all atoms in the system and constraining the total charge of the system. Consequently, for a system in which all atoms are identical (i.e., same atomic type), the computed polarization energy by PQEq would be zero for a neutral system (zero atomic charges). More details about the charge and shell position calculations by PQEq are provided in Ref. 31.

\section{B. Combination rules for the cross terms}

We propose that for the cross term $\left(\right.$ type $_{i} \neq$ type $\left._{j}\right)$ interactions between two elements, the geometric mean of the atomic parameters can be used $\left(D_{e i j}=\sqrt{ } D_{e i} D_{e j}, R_{e i j}=\sqrt{ } R_{e i} R_{e j}, L_{i j}=\sqrt{ } L_{i} L_{j}\right)$. To check the validity of this approximation, we computed the heat of vaporization $\left(\Delta H_{\text {vap }}\right)$ of noble gas crystals $\left(\mathrm{A}_{\mathrm{n}} \mathrm{B}_{\mathrm{m}}\right)$ having different stoichiometric ratios of the atomic types $(\mathrm{m}: \mathrm{n}=1: 3)$ using DFT-D3 and RexPoN. The results for the most stable volumes are provided in Table IV. The values computed by RexPoN are in reasonable agreement with DFT-D, showing that the geometric mean provides accurate cross term parameters.

For mixed element systems calculations, we must include the PQEq electrostatics energy. Here, we used the original PQEq parameters for noble gases. ${ }^{31}$ The small differences between the values in Table IV may arise from the use of the original PQEq parameters of noble gases, which have not yet been optimized using electric dipole scans.

\section{Comparison of atomic parameters with UFF values}

We compare the atomic parameters $R_{e}$ and $D_{e}$ derived from two body curves [Eq. (1)] with the UFF parameters ${ }^{13}$ in Fig. 6. In general, the $D_{e}$ values of RexPoN follow a better trend across the rows and columns of periodic tables. Also, the $D_{e}$ values of RexPoN are generally larger than UFF values (see the trends for halogens

TABLE IV. Comparison of heat of vaporization, $\Delta H_{\text {vap }}(\mathrm{kcal} / \mathrm{mol})$, of noble gas crystals $\left(A_{n} B_{m}\right)$ with various ratios of the atomic types $(m, n=1,3)$. Geometric mean average of the atomic parameters is used for cross term interactions.

\begin{tabular}{lcc}
\hline \hline & QM $\Delta \mathrm{H}_{\text {vap }}$ & $\operatorname{RexPoN} \Delta \mathrm{H}_{\text {vap }}$ \\
\hline $\mathrm{He}_{1} \mathrm{Ne}_{3}$ & -0.73 & -0.78 \\
$\mathrm{He}_{3} \mathrm{Ne}_{1}$ & -0.52 & -0.64 \\
$\mathrm{Ne}_{1} \mathrm{Ar}_{3}$ & -1.56 & -1.58 \\
$\mathrm{Ne}_{3} \mathrm{Ar}_{1}$ & -1.01 & -1.30 \\
$\mathrm{Ar}_{1} \mathrm{Kr}_{3}$ & -2.45 & -2.11 \\
$\mathrm{Ar}_{3} \mathrm{Kr}_{1}$ & -2.15 & -1.90 \\
$\mathrm{Kr}_{1} \mathrm{Xe}_{3}$ & -3.31 & -2.72 \\
$\mathrm{Kr}_{3} \mathrm{Xe}_{1}$ & -2.99 & -2.39 \\
$\mathrm{Xe}_{1} \mathrm{Rn}_{3}$ & -4.31 & -4.64 \\
$\mathrm{Xe}_{3} \mathrm{Rn}_{1}$ & -4.02 & -4.27 \\
\hline \hline
\end{tabular}




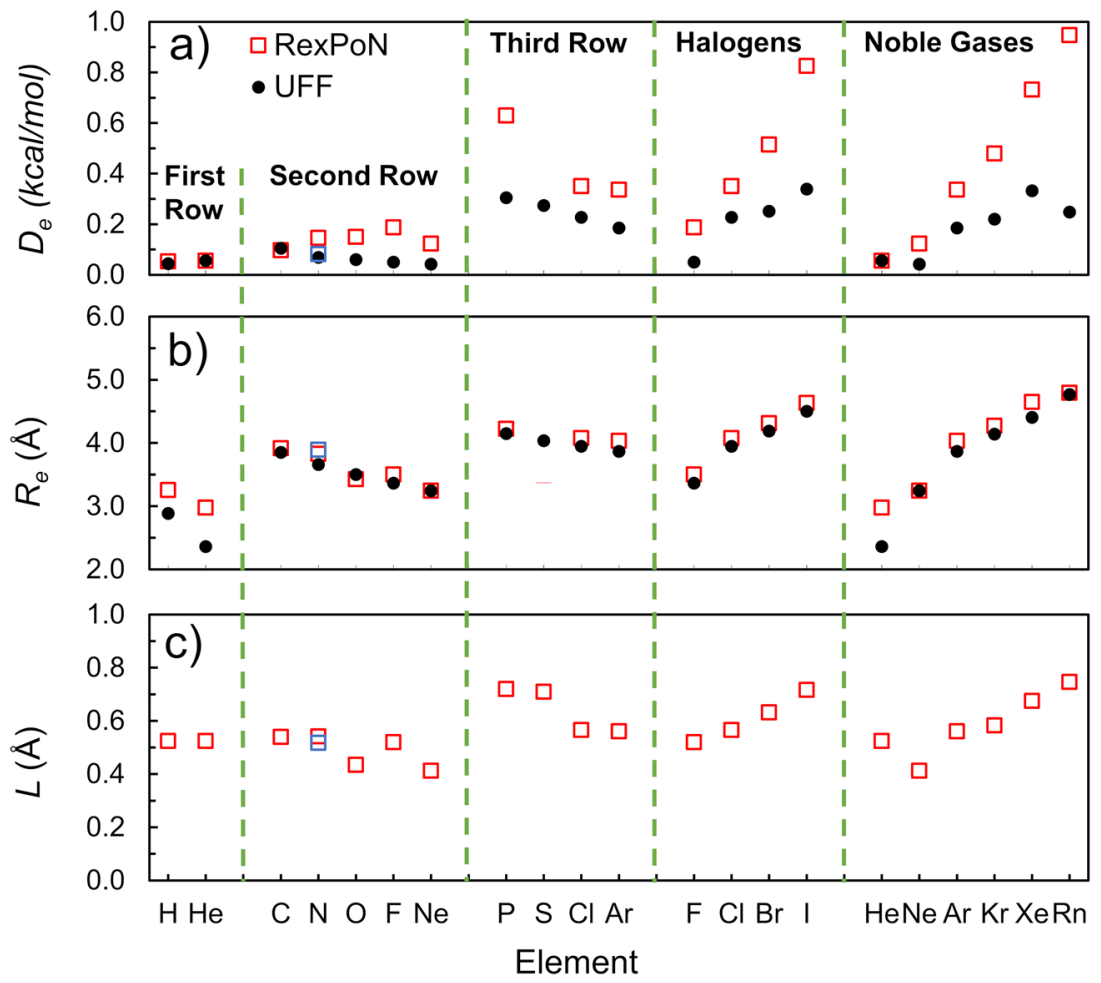

FIG. 6. The trend of $E_{U N B}$ potential atomic parameters for (a) $D_{e}$, (b) $R_{e}$, and (c) $L$ across the rows and columns of the periodic table. We compare the values of $D_{e}$ and $R_{e}$ with the UFF numbers. For nitrogen atoms, two series of values are provided for alpha- $\mathrm{N}_{2}$ (red) and for nitrogen crystals with black-phosphorus type structure (blue). and noble gases). We find the $R_{e}$ values of RexPoN and UFF to be close.

The dimensionless curvature of the $E_{U N B}$ function $\left(\omega_{U N B}^{*}\right)$ is inversely proportional to $L,\left(\omega_{U N B}^{*} \propto 1 / L^{2}\right)$. See Sec. III of the supplementary material for more details. UFF uses both the 12 6 Lennard-Jones (LJ12-6) potential and exponential 6 functions (exp6) for the vdW potential. Here, LJ12-6 leads to $\omega_{L J 12-6}^{*}=72$, whereas the curvature is an additional parameter for exp6. In UFF, optimized values were used for some atoms of the first two rows of the periodic table, but $\omega_{L J 12-6}^{*}=72$ was used for the other elements.

However, for RexPoN, $\omega_{U N B}^{*}$ differ from one atom type to another. This gives the universal nonbonded function of RexPoN flexibility to define a more accurate vdW interaction for each element type.

In Sec. III of the supplementary material, we provide a detailed comparison of $E_{U N B}$ potential with other well-known potentials including LJ12-6, Lennard-Jones 9-6 (LJ9-6), Morse, and the modified Buckingham exponential-6 (Be-6). In general, we find that the $E_{U N B}$ is softer than LJ12-6 and LJ9-6 but stiffer than Morse and Be-6 potentials.

As mentioned earlier, we used two structures to determine the atomic parameters of nitrogen. We find that the $D_{e}$ value of nitrogen with alpha structure is larger than the black-phosphorus structure, while the $R_{e}$ and $L$ values are quite close to each other.

The above comparisons suggest that for elements not studied here, we can start with the UFF atomic parameters (available up to lawrencium, $\mathrm{Z}=103$ ) for the $E_{U N B}$ function of RexPoN. However, DFT-D3 calculations should be performed for periodic cells of different atomic types and their combinations to develop accurate atomic parameters. The values of $L$ can be extrapolated or interpolated across the rows or columns to provide a reasonable value for the elements not given here.
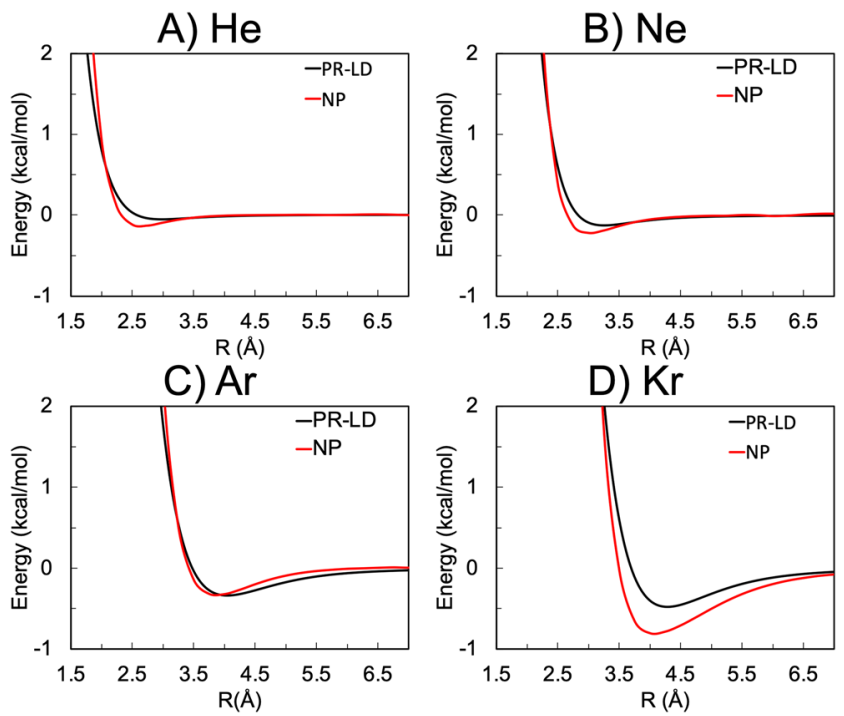

FIG. 7. The two-body nonbonded potential energy curves of noble gas elements: (a) $\mathrm{He}$, (b) $\mathrm{Ne}$, (c) Ar, and (d) $\mathrm{Kr}$ derived from EOS (ERR-LD) calculations on periodic system (black line) and directly by the scan of two atoms in a nonperiodic system $\left(\mathrm{E}_{N P}\right)(\mathrm{red})$ 


\section{Periodic versus nonperiodic calculations}

Rather than using the noble gas crystal, the noble gas pairwise nonbonded potentials can be determined from DFT-D3 on the noble gas diatomics. Figure 7 compares the two-body energy curves derived to fit the crystal EOS calculations with the diatomics computed using PBE-D3 energy with the cc-pVQZ(-g)++ basis set in the Jaguar software (see Sec. I of the supplementary material). We find that the true two body interactions are deeper at shorter distances compared to those derived from the EOS. The differences arise because the QM on the crystal includes 3-body vdW interactions (Axilrod-Teller) ${ }^{56}$ that are repulsive. Thus, fitting the EOS to two-body interactions builds in some 3-body repulsion into the two-body interactions. We consider this to be useful in condensed systems where 3-body vdW interactions exist for all the atoms.

\section{APPLICATIONS}

In this section, we use the RexPoN model for three applications to validate its accuracy. The first application uses the $E_{P R-L D}$ nonbonded term of RexPoN to compute the interfacial surface tension of liquid water at ambient temperature. The second and third applications use $E_{U N B}$ of RexPoN for five phases of the Cl-20 energetic material and nelfinavir biological system.

\section{A. Interfacial surface tension of water}

We first summarize the RexPoN FF for pure water. ${ }^{23}$ RexPoN leads to the most accurate properties ever predicted by a FF for water even though no empirical data were used. Thus, at $298 \mathrm{~K}$, RexPoN predicts ${ }^{23}$ heat of vaporization $10.36 \mathrm{kcal} / \mathrm{mol}(\exp .=10.52)$, density $0.9965 \mathrm{~g} / \mathrm{cm}^{3}$ (exp. $\left.=0.9965\right)$, entropy $68.4 \mathrm{~J} / \mathrm{mol} / \mathrm{K}$ (exp. =69.9), dielectric constant 76.1 (exp. = 78.4), and melting point $273.3 \mathrm{~K}$ (exp. = 273.15). RexPoN also reproduces the oxygen-oxygen radial distribution of the first coordination shell from neutron and $\mathrm{X}$-ray scattering very accurately. Indeed, even DFT calculations do not reproduce this description of the first coordination shell of water.

Recently, we reported that MD simulations using the RexPoN reactive $\mathrm{FF}$ at $298 \mathrm{~K}$ find an average of 2.1 Strong Hydrogen Bonds (SHBs) with an average lifetime of $\tau_{\mathrm{SHB}}=90 \mathrm{fs} .{ }^{22} \mathrm{We}$ also reported that connecting these SHBs leads to a $1 \mathrm{D}$ polymer with occasional branches to sidechains. This established the revolutionary new DynPol paradigm for the structure of water as a dynamic polydisperse branched polymer, leading to an average cluster size of 151 at $298 \mathrm{~K}$ that decreases to 36 at $400 \mathrm{~K}$. The average $\Sigma$ SHB decreases from 2.1 down to 1.6 at $400 \mathrm{~K}$, while $\tau_{S H B}$ decreases from 93 to 68 fs. The details of these simulations are described in Ref. 22.

As another indication of accuracy of our water force field, we computed the interfacial surface tension of water $\left(\gamma_{s}\right) . \gamma_{s}$ can be defined based on the free energy change that is required to change the surface area $(A)$ of air-water interface by one unit when the system is under thermodynamic equilibrium (i.e., constant temperature $T$, pressure $P$, and number of molecules $N$ ),

$$
\gamma_{s}=\left(\frac{\partial G}{\partial A}\right)_{N, T, P}=\left(\frac{G_{s}-G_{b}}{\partial A}\right)_{N, T, P},
$$

where $G_{s}$ and $G_{b}$ are the Gibbs free energy of the surface and bulk, respectively. This equation can be written based on entropy $(S)$ and surface enthalpy $(H)$ using Helmholtz free energy $(A=U-T S)$ and Gibbs-Helmholtz $(G=A+P V)$ equations,

$$
\gamma_{s}=\left(\frac{H_{s}-H_{b}+T S_{s}-T S_{b}}{\partial A}\right)_{N, T, P}=\left(\frac{\Delta h_{e x}-T \Delta s_{e x}}{\partial A}\right),
$$

where $h_{e x}$ is molar excess surface enthalpy and $s_{e x}$ is molar excess surface entropy.

To compute $\gamma_{s}$ based on Eq. (10), we use the two-phase thermodynamics (2PT) methodology. ${ }^{57,58}$ 2PT allows for the direct calculation of absolute thermodynamics of the system based on the Fourier transform of the velocity autocorrelation function. The accuracy of $2 \mathrm{PT}$ for the calculation of thermodynamics of $2 \mathrm{D}$ slab geometries has been validated before. ${ }^{5}$

We computed $\gamma_{s}$ of liquid water by performing two series of calculations. For the first calculation, we utilized bulk water simulations at $298 \mathrm{~K}$ for a system with 432 molecules per cell. We first equilibrated the system at $298 \mathrm{~K}$ by running $0.6 \mathrm{~ns}$ constant volume, constant temperature (NVT), molecular dynamics (MD) simulations. To control the temperature, we used the Langevin thermostat. ${ }^{60}$ After this initial equilibration, we utilized $1.1 \mathrm{~ns} \mathrm{MD}$ simulation using constant pressure (1 atm), constant temperature (NPT) ensemble to fully relaxed the simulation cell. Finally, we further equilibrated the system at $298 \mathrm{~K}$ for another $0.6 \mathrm{~ns}$ using NVT ensemble simulations. We saved the coordinates and velocities from the last 100 ps trajectories ( 5 cycles each 20 ps) every 1 fs to perform 2PT calculations and compute $\gamma_{\mathrm{s}}$.

For the second calculation, we started from the equilibrated bulk coordinates and velocities and performed 2D slab (surface)

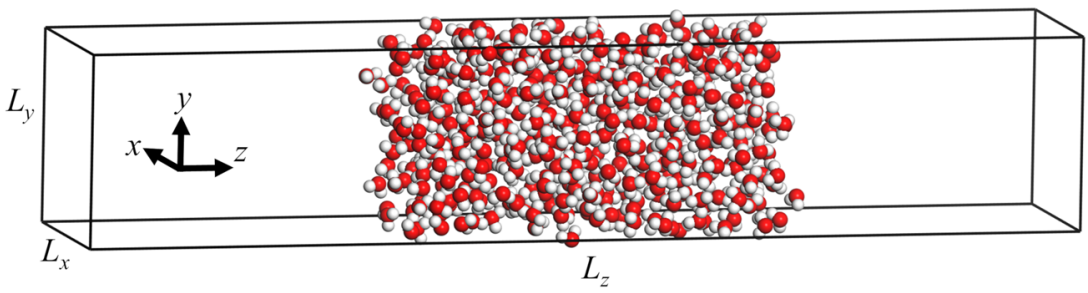

FIG. 8. Snapshot of the air-water simulation cell showing 432 equilibrated water molecules at $298 \mathrm{~K}$. The dimensions of the cell are $L_{x}=L_{y}=1.86 \mathrm{~nm}$, and $L_{z}=9.70 \mathrm{~nm}$. There is about $3 \mathrm{~nm}$ of vacuum on each surface in the $z$ direction. The surface was relaxed at $298 \mathrm{~K}$ for about $2 \mathrm{~ns}$ using MD-NVT simulations with RexPoN FF. 
simulations at $298 \mathrm{~K}$. We inflated the simulation cell of the bulk system in the $z$ direction by about $6 \mathrm{~nm}$ and centered the slab within the cell allowing about $3 \mathrm{~nm}$ of vacuum at each surface of liquid (see Fig. 8). We then performed NVT simulations of the surface at $298 \mathrm{~K}$ for about 2 ns to equilibrate the system. Similar to bulk simulations, we used the trajectories from the last 100 ps to perform $2 \mathrm{PT}$ calculations.

For all of the above simulations, we used the RexPoN rigid water model ${ }^{23}$ with the time step of 1.0 fs. To control the pressure, we used a barostat with a relaxation time of $2 \mathrm{ps}$, and to control temperature, we used damping time of $100 \mathrm{fs}$.

We used 2PT to compute interfacial surface tension $\left(\gamma_{s}\right)$ according to Eq. (9) as

$$
\gamma_{s}=c \frac{G_{s}-G_{b}}{S A},
$$

where $\mathrm{c}=166.03$ is the conversion which gives $\gamma_{s}$ in dynes per centimeter and SA is the total surface area of both surfaces $(S A=2 \times$ $\left.L_{x} \times L_{y}\right)$. More details about the methodology to calculate interfacial surface tension using 2PT are provided in Ref. 59.

Table $\mathrm{V}$ compares the experimental and computed interfacial surface tension $\left(\gamma_{s}\right)$ of liquid water using RexPoN and some of the famous water models including TIP $4 \mathrm{P} / 2005,{ }^{4} \mathrm{~mW},{ }^{5} \mathrm{SPC} / \mathrm{Ew},{ }^{6}$ and TIP3P. $\gamma_{s}$ for these water models were computed by $2 \mathrm{PT}$ method, and the data were taken from Ref. 59. As shown, the RexPoN computed $\gamma_{s}=69.5 \mathrm{dyn} / \mathrm{cm}$ is in great agreement with the experimental value of $71.9 \mathrm{dyn} / \mathrm{cm}$.

\section{B. High energy materials and composites}

It is crucial to predict the crystal structure of new energetic materials (EMs) worthy of synthesis and characterization prior to the experiment. ${ }^{62,63}$ EM have widespread applications in many areas including space exploration and mining. Predicting reliably the
TABLE V. Comparison of experimental ${ }^{61}$ and computed interfacial surface tension $\left(\gamma_{s}, \mathrm{dyn} / \mathrm{cm}\right)$ of liquid water at $298 \mathrm{~K}$. The computed $\gamma_{s}$ for TIP4P/2005, mW, SPC/Ew, and TIP3P are from Ref. 59.

\begin{tabular}{ccccccc}
\hline & Expt. & RexPoN & TIP4P/2005 & $\mathrm{mW}$ & SPC/Ew & TIP3P \\
\hline$\gamma_{s}(\mathrm{dyn} / \mathrm{cm})$ & 71.9 & 69.5 & 64.4 & 66.9 & 55.8 & 52.6 \\
\hline
\end{tabular}

constitutive and chemical properties, as well as initiation kinetics processes that control stability, performance, and sensitivity prior to synthesis and characterization is critical to the development of novel high-energy density materials (HEDMs) and high-energy dense oxidizers (HEDOs).

The current classical FF with parameters fitted to empirical data are not sufficiently accurate for guiding the development of next generation EMs (see below), which may exhibit quite different crystal, constitutive, and reaction kinetics properties. To evaluate the accuracy of the RexPoN nonbond potential for different EM polymorphs, we choose a well-studied energetic crystal, hexanitro-hexaaza-isowurtzitane (CL-20), for which five stable crystal polymorphs are known experimentally: ${ }^{65-67}$ the $\alpha, \beta, \varepsilon, \gamma$, and $\xi$ phases shown in Fig. 9.

First, we optimized the periodic crystal cell using PBE-D3 DFT to obtain the most stable structures within each phase. The dispersion-corrected DFT methods have been tested previously to predict experimental crystal structures and densities of EM to $1.6 \%-$ $2.1 \%$ maximum error for the lattice parameters. ${ }^{68}$ After optimization, we estimated the sublimation energy at $\mathrm{T}=0 \mathrm{~K}\left(\Delta_{0 K} H_{s u b}\right)$ for each crystal phase by subtracting the total energy of the crystal from the energy of reference molecule outside of the corresponding cell. In the case of the $\alpha$-phase structure, the reference molecules are the $\alpha-$ CL-20 and water molecule. For other phases,
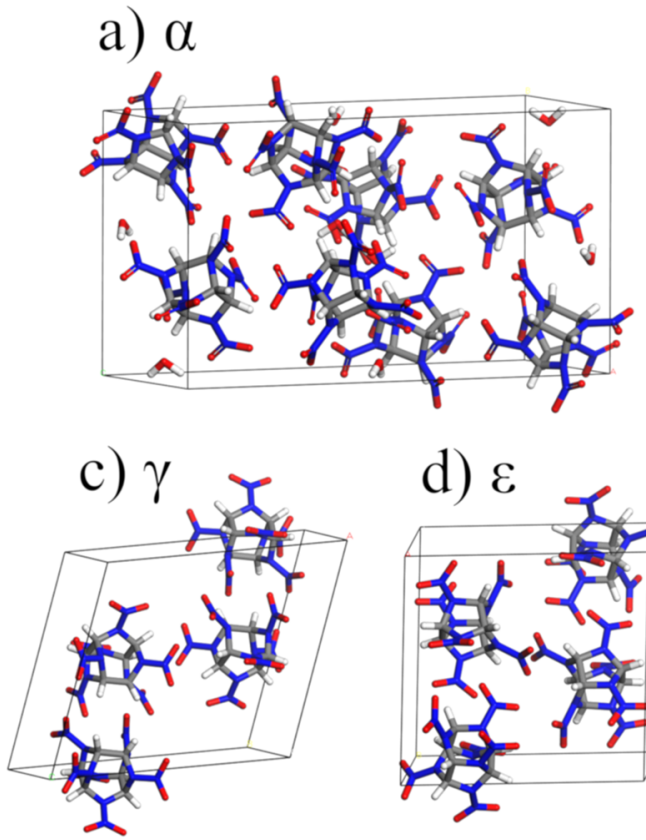

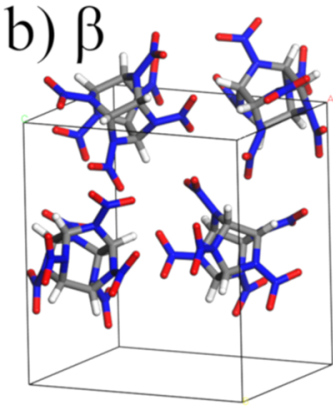

d) $\varepsilon$

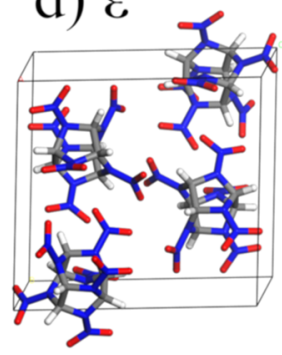

e) $\xi$

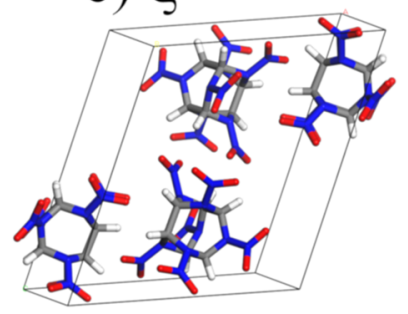

FIG. 9. The crystal structure of (a) $\alpha$-, (b) $\beta-$, (c) $\gamma$-, (d) $\varepsilon$-, and (e) $\xi$-phases of CL20 energetic material. 
the reference molecule is taken from the corresponding phase of CL-20 crystal. Next, we calculated $\Delta_{0 K} H_{\text {sub }}$ using the RexPoN FF for the same crystal structures obtained from the PBE-D3 DFT optimization.

Here, the nonbonded interactions consist of vdW and electrostatic (including polarization) energies. For electrostatics, we used the PQEq model with the parameters optimized based on the electric dipole energy scans for cyclohexane-based molecular structures. ${ }^{3 .}$ No EM molecules or structures were used to optimize the PQEq parameters. To provide a better understanding about the accuracy level of the RexPoN FF, we also computed $\Delta_{0 K} H_{\text {sub }}$ for each of the above phases using the OPLS ${ }^{69}$ FF developed to study biomolecular and condensed matter systems. We used the parameters from OPLS2005 $\mathrm{FF}^{70}$ generated from the Desmond system builder.

The results are compared in Table VI. As shown, the RexPoN $\Delta_{0 K} H_{s u b}$ values for all phases are in much better agreement with the DFT-D3 calculations than OPLS FF. For the nitrogen parameters of RexPoN, we used the N parameters based on the black phosphorus structure, $\mathrm{N}(\mathrm{BP})$, since it has bonding more similar to the $\mathrm{N}$ atoms in CL-20.

In terms of the ranking order for the heat of sublimation at $0 \mathrm{~K}, \mathrm{RexPoN}$ and PBE-D3 find hydrated $\alpha$-phase of CL-20 to be the most stable by $\Delta_{0 \mathrm{~K}} \mathrm{H}_{\text {sub }}=-47.2$ and $-48.7 \mathrm{kcal} / \mathrm{mol}$, respectively. The $\Delta_{0 K} H_{\text {sub }}$ value of RexPoN for the least stable $\xi$ phase $(-33.59 \mathrm{kcal} / \mathrm{mol})$ is also quite close to the DFT-D value $(-33.05 \mathrm{kcal} / \mathrm{mol})$. For the $\beta, \gamma$, and $\varepsilon$ phases, both DFT and RexPoN find them within a range of $3 \mathrm{kcal} / \mathrm{mol}$ but slightly different ordering. This could be due to the fact that PQEq parameters were not optimized for energetic materials. Although no direct experimental measurements of the heat of sublimation for CL-20 polymorphs have been published, our results are in reasonable agreement with available experiment-based estimates $(35.21-40.51 \mathrm{kcal} / \mathrm{mol})$ for the sublimation on burning surface ${ }^{72}$ and theoretical calculations (37.52 $\pm 4.8 \mathrm{kcal} / \mathrm{mol}^{73}$ and $40.32 \mathrm{kcal} / \mathrm{mol}^{74}$ ). $\mathrm{kcal} / \mathrm{mol}$.

In contrast, $\Delta_{0 \mathrm{~K}} H_{\text {sub }}$ from OPLS are too large by 11-23

\section{Biological systems}

A major challenge in rational drug design is the accurate prediction of drug-target interactions, which are predominantly noncovalent in nature.

Accurate QM-based methods are not practical since a protein with 400 amino acids, including water and ions, may lead to 30000 atoms. The available classical FFs are not generally sufficiently accurate to obtain the desired accuracy for various druglike molecules. One reason is that these FFs are often optimized for particular

TABLE VI. Heat of sublimation at $0 \mathrm{~K}, \Delta_{O K} H_{\text {sub }}(\mathrm{kcal} / \mathrm{mol})$, of various phases of $\mathrm{CL}-20$ calculated with PBE-D3, RexPoN, and OPLS 2005 FF.

\begin{tabular}{llll}
\hline \hline Phases & PBE-D3 & RexPoN & OPLS 2005 \\
\hline$\alpha$ & -48.72 & -47.22 & -65.64 \\
$\beta$ & -35.63 & -36.33 & -52.54 \\
$\gamma$ & -37.06 & -33.17 & -48.12 \\
$\varepsilon$ & -35.52 & -35.32 & -54.10 \\
$\xi$ & -33.05 & -33.59 & -55.91 \\
\hline \hline
\end{tabular}

systems since it is difficult to find parameters fitting a wide range of compounds (due to huge dimensionality of the chemical space). In addition, these nonbonded interactions directly affect the thermodynamics properties of the system including enthalpy, free energy, and entropy which have special importance in structural biology. ${ }^{76}$ It has been a daunting challenge to accurately describe the thermodynamics of these nonbonded interactions at the molecular level. $^{76}$

We demonstrate here that RexPoN FF can accurately predict the binding energies of water molecules against the nelfinavir structure (an HIV type-1 protease inhibitor). Here, we first calculated the binding energy, $\Delta E_{\text {bind }}=E_{\text {total }}-\left(E_{\text {nelfinavir }}+E_{\text {water }}\right)$, of a single molecule of water to nelfinavir for several binding sites (see Fig. 10) using QM. The nonperiodic structure of nelfinavir was initially optimized in QM (PBE-D3 DFT) using the $6-31 \mathrm{G}^{* *}$ basis set. ${ }^{77,78} \mathrm{We}$ then introduced a molecule of the water to the system and optimized the water using QM, while keeping nelfinavir fixed. Next, we computed $\Delta E_{\text {bind }}$ using RexPoN and Amber03 FFs. ${ }^{80}$ For the RexPoN calculations, we started by just including the PQEq polarization and vdW nonbonded $\left(E_{U N B}\right)$ energy terms. For PQEq, we used parameters optimized based on the electric dipole energy scans for cyclohexane-based molecular structures. ${ }^{31}$ For the atomic parameters of sulfur, we used a linear interpolation of atomic parameters of the elements from the same row (i.e., $\mathrm{P}, \mathrm{Cl}$, and Ar). For nitrogen, we used the $\mathrm{N}$ (BP) parameters. For the parameters of water in Amber03 FF, we used SPC water.

The results of binding energy calculations are summarized in Table VII. As shown, $\Delta E_{\text {bind }}$ of RexPoN is in reasonable agreement with $\Delta E_{b i n d}$ of QM with the root-mean-square error (RMSE) of $2.4 \mathrm{kcal} / \mathrm{mol}$, much better than $\Delta E_{\text {bind }}$ of Amber03 (RMSE $=6.1 \mathrm{kcal} / \mathrm{mol}$ ). These results show that the PQEq model is able to account for most of short-range hydrogen bond (HB) contributions that are important in such polar systems.

However, it is well known that proteins need special HB corrections to account for the very polar nature of the amino acids. Thus, we considered an explicit term for the short-range $\mathrm{HB}$ interactions. For nelfinavir, these $\mathrm{HB}$ interactions are between water and oxygen (sites 1-5), nitrogen (site 9), and sulfur (site 8), as shown in Fig. 10.

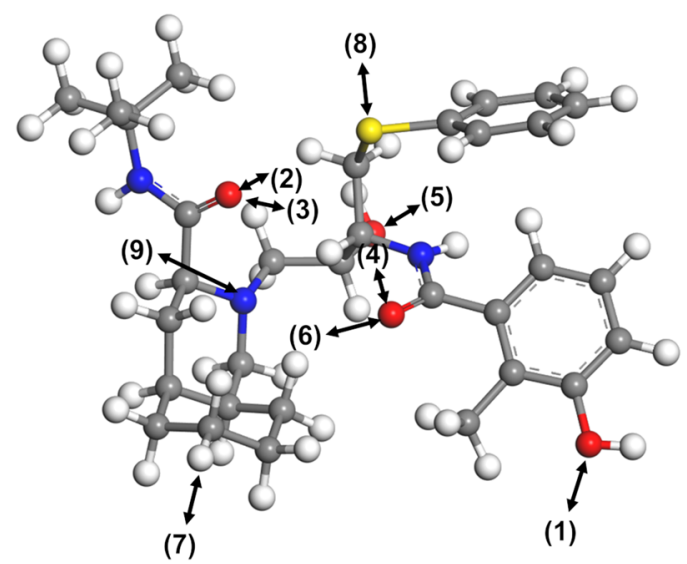

FIG. 10. Binding sites of the water molecule to the nelfinavir structure. The sites were selected to also study the hydrogen bond effects on the binding energies. 
TABLE VII. Binding energy $\left(\Delta E_{\text {bind }}, \mathrm{kcal} / \mathrm{mol}\right)$ of the water molecule to the various sites (shown in Fig. 10) of the nelfinavir structure using QM, RexPoN, and Amber03 FFs with and without the HB $\left(E_{H B}\right)$ corrections [Eq. (12)]. The RMSE $(\mathrm{kcal} / \mathrm{mol})$ is calculated for all of the binding sites in each column.

\begin{tabular}{lccccc}
\hline Site & QM & RexPoN & Amber03 & RexPoN + HB & Amber03 + HB \\
\hline 1 & -8.73 & -3.12 & -3.13 & -8.46 & -8.47 \\
2 & -11.16 & -6.87 & 2.08 & -12.43 & -3.47 \\
3 & -12.77 & -8.77 & 0.11 & -15.20 & -6.32 \\
4 & -15.66 & -9.96 & 0.32 & -17.89 & -7.61 \\
5 & -16.63 & -4.41 & 16.45 & -14.52 & 6.34 \\
6 & -20.82 & -8.31 & 0.61 & -19.89 & -10.97 \\
7 & -7.04 & -3.75 & 0.90 & -6.03 & -1.37 \\
8 & -5.55 & -4.06 & -4.48 & -5.44 & -5.85 \\
9 & 5.72 & 12.87 & 33.20 & 7.25 & 27.59 \\
RMSE & $\ldots$ & 2.4 & 6.1 & 0.5 & 4.0 \\
\hline \hline
\end{tabular}
given by

We use a similar Morse-type $\mathrm{HB}$ term as in Dreiding $\mathrm{FF}^{15,79}$

$$
E_{H B}(r)=D_{h b}\left\{\exp \left[-2 \alpha_{h b}\left(r-r_{h b}\right)\right]-2 \exp \left[-\alpha_{h b}\left(r-r_{h b}\right)\right]\right\} \cos ^{n_{h b}} \theta,
$$

where $D_{h b}, \alpha_{h b}, r_{h b}$, and $n_{h b}$ are the parameters to be determined. Our goal is to have only one set of global HB parameters that can work well for any kind of $\mathrm{HB}$ interactions such as the ones given in Fig. 10. To do this, we started with Dreiding $\mathrm{HB}$ energy and distance values $\left(D_{h b}=9.5 \mathrm{kcal} / \mathrm{mol}\right.$ and $\left.r_{h b}=2.75^{\circ} \AA\right)$ from Ref. 15 and selected $\alpha_{h b}$ $=5 / r_{h b}=1.82$ and $n_{h b}=2$ according to Ref. 79 .

Then, we used the QM binding energy of sites 1, 8, and 9 (given in Table VII) as the training set and only modified $D_{h b}, \alpha_{h b}$, and $r_{h b}$ to get the best agreement with QM values. The optimized parameters are $D_{h b}=8.0 \mathrm{kcal} / \mathrm{mol}, \alpha_{h b}=1.72, r_{h b}=2.92 \AA$, and $n_{h b}=2$.

The results for $\Delta E_{b i n d}$ of RexPoN plus $E_{H B}$ for different binding sites are given in Table VII. We find that using these HB parameters together with PQEq polarization and vdW nonbonded $\left(E_{U N B}\right)$, energy terms provide excellent agreement between the computed RexPoN and QM (RMSE $=0.5 \mathrm{kcal} / \mathrm{mol}$ )

Amber03 FF does not include an explicit term for the calculation of $\mathrm{HB}$ energy. Instead, the $\mathrm{HB}$ interactions are captured via modified 2-body vdW plus electrostatic energy terms. We wondered whether adding the HB energy term of RexPoN would help to improve the results of Amber03 FF. In Table VII, we show that addition of the RexPoN HB term to Amber03 FF slightly improves the results, but the difference between Amber03 FF and QM remains high with the RMSE of $4.0 \mathrm{kcal} / \mathrm{mol}$, which is worse than RexPoN without $\mathrm{HB}$ corrections $(\mathrm{RMSE}=2.4 \mathrm{kcal} / \mathrm{mol}$ )

\section{CONCLUSION}

The goal of this paper is to report a new methodology aimed at defining all nonbonded components (vdW, electrostatics, hydrogen bonds) for use in force fields. These new nonbonded terms may be suitable for use in current FFs such as OPLS, AMBER, and CHARMM, but we have not tested this application because our main interest is for a new generation reactive RexPoN FF based only on accurate QM, such as the one we developed for the water system. ${ }^{22,23}$ Thus, each component of the FF is defined separately based on direct QM energies or forces. This paper focuses on developing the vdW part of the RexPoN FF, using DFT-D3 calculations including dispersion corrections to obtain the energy-volume EOS of single element crystals. The use of single element data excludes polarization and electrostatics contributions so that the DFT provides the vdW energy. In particular, we derived the vdW nonbonded potential parameters $\left(E_{P R-L D}\right)$ for $\mathrm{H}, \mathrm{C}, \mathrm{N}, \mathrm{O}, \mathrm{P}, \mathrm{F}, \mathrm{Cl}, \mathrm{Br}, \mathrm{I}, \mathrm{He}, \mathrm{Ne}, \mathrm{Ar}, \mathrm{Kr}, \mathrm{Xe}$, and $\mathrm{Rn}$, based on the DFT-D energy-volume EOS. These vdW nonbonded potentials are suitable to describe interatomic interactions for long- and short-range interactions.

In addition, we discovered that the vdW potential curves extracted from EOS for the 6 noble gases lead to essentially identical curves, $E_{U N B}$, after scaling with just 3 parameters, $D_{e}, R_{e}$, and $L$. This suggests that this universal nonbonded curve $\left(E_{U N B}\right)$ can be used for all atoms of the periodic table. This simplifies the task of obtaining vdW for the remainder of the periodic table since we need to only find ways to fit the trends in $D_{e}, R_{e}$, and $L$ along the rows or columns.

We use the PQEq model to describe polarization and electrostatics in various materials. PQEq uses Gaussian-shaped electron densities for atoms and dynamically updates the charge distribution and electron shell positions across the system. The important advantage of PQEq is that all parameters of the model are defined directly based on QM energy of electric probe dipoles with no vdW contributions. The combination of $E_{P R-L D}$ (or $E_{U N B}$ ) with PQEq defines the dominant part of the nonbonded potential of RexPoN FF. Therefore, we make no assumptions to specify the contributions of vdW, electrostatics, and other components of the nonbonded potential to the total QM energy. In principle, this should provide transferability and accuracy of RexPoN FF for new systems and materials without the need to reoptimize the parameters for specific systems being studied. We validated the accuracy of $E_{P R-L D}$ and $E_{U N B}$ for several systems including water system, energetic materials, and biological systems.

\section{SUPPLEMENTARY MATERIAL}

The supplementary material includes computational details, description of the training set, comparison between universal nonbonded potential of RexPoN and other potentials, and melting point calculations for different isotopes of water.

\section{ACKNOWLEDGMENTS}

S.N. was supported by the Joint Center for Artificial Photosynthesis, a DOE Energy Innovation Hub, supported through the Office of Science of the U.S. Department of Energy under Award No. DE-SC0004993. W.A.G. was supported by the Computational Materials Sciences Program funded by the U.S. Department of Energy, Office of Science, Basic Energy Sciences, under Award Number DESC00014607. S.Z. was supported by the Office of Naval Research Grant Nos. N00014-19-1-2081 and N00014-18-1-2155. J.J.O. was supported by the Ernest H. Swift and Arthur A. Noyes SURF Fellowships. The calculations were carried out on the Extreme Science and Engineering Discovery Environment (XSEDE), which is supported by the National Science Foundation, Grant No. ACI-1548562. 


\section{REFERENCES}

${ }^{1}$ W. L. Jorgensen, J. D. Madura, and C. J. Swenson, "Optimized intermolecular potential functions for liquid hydrocarbons," J. Am. Chem. Soc. 106(22), 66386646 (1984).

${ }^{2}$ J. Wang, R. M. Wolf, J. W. Caldwell, P. A. Kollman, and D. A. Case, "Development and testing of a general amber force field," J. Comput. Chem. 25(9), 1157-1174 (2004)

${ }^{3}$ A. D. MacKerell, D. Bashford, M. Bellott, R. L. Dunbrack, J. D. Evanseck, M. J. Field, S. Fischer, J. Gao, H. Guo, S. Ha et al., "All-atom empirical potential for molecular modeling and dynamics studies of proteins," J. Phys. Chem. B 102(18), 3586-3616 (1998).

${ }^{4} \mathrm{~J}$. L. Abascal and C. Vega, "A general purpose model for the condensed phases of water: TIP4P/2005,” J. Chem. Phys. 123(23), 234505 (2005).

${ }^{5} \mathrm{~V}$. Molinero and E. B. Moore, "Water modeled as an intermediate element between carbon and silicon," J. Phys. Chem. B 113(13), 4008-4016 (2008).

${ }^{6} \mathrm{H}$. Berendsen, J. Grigera, and T. Straatsma, "The missing term in effective pair potentials," J. Phys. Chem. 91(24), 6269-6271 (1987).

${ }^{7}$ W. L. Jorgensen, J. Chandrasekhar, J. D. Madura, R. W. Impey, and M. L. Klein, "Comparison of simple potential functions for simulating liquid water," J. Chem. Phys. 79(2), 926-935 (1983).

${ }^{8}$ S. Naserifar, L. Liu, W. A. Goddard III, T. T. Tsotsis, and M. Sahimi, “Toward a process-based molecular model of $\mathrm{SiC}$ membranes. 1. Development of a reactive force field," J. Phys. Chem. C 117(7), 3308-3319 (2013).

${ }^{9}$ S. Naserifar, W. A. Goddard III, L. Liu, T. T. Tsotsis, and M. Sahimi, "Toward a process-based molecular model of SiC membranes. 2. Reactive dynamics simulation of the pyrolysis of polymer precursor to form amorphous SiC," J. Phys. Chem. C 117(7), 3320-3329 (2013).

${ }^{10}$ A. van Duin, O. Verners, and Y.-K. Shin, "Reactive force fields: Concepts of ReaxFF and applications to high-energy materials," Int. J. Energ. Mater. Chem. Propul. 12(2), 95 (2013).

${ }^{11}$ K. D. Nielson, A. C. Van Duin, J. Oxgaard, W.-Q. Deng, and W. A. Goddard, "Development of the ReaxFF reactive force field for describing transition metal catalyzed reactions, with application to the initial stages of the catalytic formation of carbon nanotubes," J. Phys. Chem. A 109(3), 493-499 (2005).

${ }^{12}$ J. E. Mueller, A. C. van Duin, and W. A. Goddard III, "Development and validation of ReaxFF reactive force field for hydrocarbon chemistry catalyzed by nickel," J. Phys. Chem. C 114(11), 4939-4949 (2010).

${ }^{13}$ A. K. Rappe, C. J. Casewit, K. S. Colwell, W. A. Goddard, and W. M. Skiff, "UFF, a full periodic table force field for molecular mechanics and molecular dynamics simulations," J. Am. Chem. Soc. 114(25), 10024-10035 (1992).

${ }^{14}$ A. C. van Duin, S. Dasgupta, F. Lorant, and W. A. Goddard, "ReaxFF: A reactive force field for hydrocarbons,” J. Phys. Chem. A 105(41), 9396-9409 (2001).

${ }^{15}$ S. L. Mayo, B. D. Olafson, and W. A. Goddard, "DREIDING: A generic force field for molecular simulations," J. Phys. Chem. 94(26), 8897-8909 (1990).

${ }^{16}$ A. Vedani and D. W. Huhta, "A new force field for modeling metalloproteins," J. Am. Chem. Soc. 112(12), 4759-4767 (1990).

${ }^{17}$ N. L. Allinger, "Conformational analysis. 130. MM2. A hydrocarbon force field utilizing V1 and V2 torsional terms," J. Am. Chem. Soc. 99(25), 8127-8134 (1977).

${ }^{18}$ N. L. Allinger, Y. H. Yuh, and J. H. Lii, "Molecular mechanics. The MM3 force field for hydrocarbons. 1," J. Am. Chem. Soc. 111(23), 8551-8566 (1989).

${ }^{19} \mathrm{O}$. Borodin, "Polarizable force field development and molecular dynamics simulations of ionic liquids," J. Phys. Chem. B 113(33), 11463-11478 (2009).

${ }^{20}$ T. A. Halgren, "Merck molecular force field. I. Basis, form, scope, parameterization, and performance of MMFF94," J. Comput. Chem. 17(5-6), 490-519 (1996).

${ }^{21}$ J. W. Ponder, C. Wu, P. Ren, V. S. Pande, J. D. Chodera, M. J. Schnieders, I. Haque, D. L. Mobley, D. S. Lambrecht, R. A. DiStasio et al., "Current status of the AMOEBA polarizable force field," J. Phys. Chem. B 114(8), 2549-2564 (2010).

${ }^{22}$ S. Naserifar and W. A. Goddard, "Liquid water is a dynamic polydisperse branched polymer," Proc. Natl. Acad. Sci. 116(6), 1998-2003 (2019).

${ }^{23}$ S. Naserifar and W. A. Goddard III, "The Quantum mechanics-based polarizable force field for water simulations," J. Chem. Phys. 149(17), 174502 (2018).
${ }^{24}$ G. R. Medders, V. Babin, and F. Paesani, "Development of a "First-Principles" water potential with flexible monomers. III. Liquid phase properties," J. Chem. Theory Comput. 10(8), 2906-2910 (2014).

${ }^{25}$ R. Bukowski, K. Szalewicz, G. C. Groenenboom, and A. van der Avoird, "Polarizable interaction potential for water from coupled cluster calculations. I. Analysis of dimer potential energy surface," J. Chem. Phys. 128(9), 094313 (2008).

${ }^{26}$ J. W. Ponder and D. A. Case, "Force fields for protein simulations," in Advances in Protein Chemistry (Elsevier, 2003), Vol. 66, pp. 27-85.

${ }^{27}$ S. Cox and D. Williams, "Representation of the molecular electrostatic potential by a net atomic charge model," J. Comput. Chem. 2(3), 304-323 (1981).

${ }^{28}$ D. E. Williams, "Representation of the molecular electrostatic potential by atomic multipole and bond dipole models," J. Comput. Chem. 9(7), 745-763 (1988).

${ }^{29}$ L. C. Cusachs and P. Politzer, "On the problem of defining the charge on an atom in a molecule," Chem. Phys. Lett. 1(11), 529-531 (1968).

${ }^{30}$ P.-O. Löwdin, "On the nonorthogonality problem," in Advances in Quantum Chemistry (Elsevier, 1970), Vol. 5, pp. 185-199.

${ }^{31}$ S. Naserifar, D. J. Brooks, W. A. Goddard, and V. Cvicek, "Polarizable charge equilibration model for predicting accurate electrostatic interactions in molecules and solids," J. Chem. Phys. 146(12), 124117 (2017).

${ }^{32}$ J. J. Oppenheim, S. Naserifar, and W. A. Goddard III, "Extension of the polarizable charge equilibration model to higher oxidation states with applications to $\mathrm{Ge}, \mathrm{As}, \mathrm{Se}, \mathrm{Br}, \mathrm{Sn}, \mathrm{Sb}, \mathrm{Te}, \mathrm{I}, \mathrm{Pb}, \mathrm{Bi}, \mathrm{Po}$, and at elements," J. Phys. Chem. A 122(2), 639-645 (2018).

${ }^{33}$ S. Kwon, S. Naserifar, H. M. Lee, and W. A. Goddard, "Polarizable charge equilibration model for transition-metal elements," J. Phys. Chem. A 122(48), 9350-9358 (2018).

${ }^{34}$ S. Grimme, "Semiempirical GGA-type density functional constructed with a long-range dispersion correction," J. Comput. Chem. 27(15), 1787-1799 (2006).

${ }^{35}$ S. Grimme, J. Antony, S. Ehrlich, and H. Krieg, "A consistent and accurate ab initio parametrization of density functional dispersion correction (DFT-D) for the 94 elements H-Pu," J. Chem. Phys. 132(15), 154104 (2010).

${ }^{36}$ P. Loubeyre, R. LeToullec, D. Hausermann, M. Hanfland, R. Hemley, H. Mao, and L. Finger, "X-ray diffraction and equation of state of hydrogen at megabar pressures," Nature 383(6602), 702 (1996).

${ }^{37} \mathrm{~J}$. Kohanoff, S. Scandolo, G. L. Chiarotti, and E. Tosatti, "Solid molecular hydrogen: The broken symmetry phase," Phys. Rev. Lett. 78(14), 2783 (1997).

${ }^{38}$ M. Städele and R. M. Martin, "Metallization of molecular hydrogen: Predictions from exact-exchange calculations," Phys. Rev. Lett. 84(26), 6070 (2000).

${ }^{39}$ C. Barrett, L. Meyer, and J. Wasserman, "Antiferromagnetic and crystal structures of alpha-oxygen,” J. Chem. Phys. 47(2), 592-597 (1967).

${ }^{40}$ Y. A. Freiman and H.-J. Jodl, "Solid oxygen," Phys. Rep. 401(1-4), 1-228 (2004).

${ }^{41}$ S. Somiya, Handbook of Advanced Ceramics: Materials, Applications, Processing, and Properties (Academic Press, 2013).

${ }^{42}$ E. Y. Tonkov and E. Ponyatovsky, Phase Transformations of Elements Under High Pressure (CRC Press, 2004).

${ }^{43} \mathrm{~A}$. Brown and S. Rundqvist, "Refinement of the crystal structure of black phosphorus," Acta Crystallogr. 19(4), 684-685 (1965).

${ }^{44}$ L. Meyer, C. Barrett, and S. C. Greer, "Crystal structure of a-fluorine," J. Chem. Phys. 49(4), 1902-1907 (1968).

${ }^{45} \mathrm{~B}$. Powell, K. Heal, and B. Torrie, "The temperature dependence of the crystal structures of the solid halogens, bromine and chlorine," Mol. Phys. 53(4), 929-939 (1984).

${ }^{46}$ R. Ibberson, O. Moze, and C. Petrillo, "High resolution neutron powder diffraction studies of the low temperature crystal structure of molecular iodine (I2)," Mol. Phys. 76(2), 395-403 (1992).

${ }^{47}$ G. L. Pollack, "The solid state of rare gases," Rev. Mod. Phys. 36(3), 748 (1964). ${ }^{48}$ A. D. Becke, "Density-functional Thermochemistry. III. The role of exact exchange," J. Chem. Phys. 98(7), 5648-5652 (1993).

${ }^{49}$ C. Lee, W. Yang, and R. G. Parr, "Development of the colle-salvetti correlationenergy formula into a functional of the electron density," Phys. Rev. B 37(2), 785 (1988). 
${ }^{50}$ S. Grimme, S. Ehrlich, and L. Goerigk, "Effect of the damping function in dispersion corrected density functional theory," J. Comput. Chem. 32(7), 1456-1465 (2011).

${ }^{51}$ Y. Liu and W. A. Goddard III, "First-principles-based dispersion augmented density functional theory: From molecules to crystals," J. Phys. Chem. Lett. 1(17), 2550-2555 (2010).

${ }^{52}$ H. Kim, J.-M. Choi, and W. A. Goddard III, "Universal correction of density functional theory to include London dispersion (up to Lr, element 103)," J. Phys. Chem. Lett. 3(3), 360-363 (2012).

${ }^{53}$ J. H. Rose, J. R. Smith, and J. Ferrante, "Universal features of bonding in metals," Phys. Rev. B 28(4), 1835 (1983).

${ }^{54}$ Y. P. Varshni, "Comparative study of potential energy functions for diatomic molecules,” Rev. Mod. Phys. 29(4), 664 (1957).

${ }^{55}$ S. Plimpton, "Fast parallel algorithms for short-range molecular dynamics," J. Comput. Phys. 117(1), 1-19 (1995).

${ }^{56} \mathrm{~B}$. Axilrod and E. Teller, "Interaction of the van der Waals type between three atoms," J. Chem. Phys. 11(6), 299-300 (1943).

${ }^{57}$ S.-T. Lin, P. K. Maiti, and W. A. Goddard III, "Two-phase thermodynamic model for efficient and accurate absolute entropy of water from molecular dynamics simulations," J. Phys. Chem. B 114(24), 8191-8198 (2010).

${ }^{58}$ T. A. Pascal, S.-T. Lin, and W. A. Goddard III, "Thermodynamics of liquids: Standard molar entropies and heat capacities of common solvents from 2PT molecular dynamics," Phys. Chem. Chem. Phys. 13(1), 169-181 (2011).

${ }^{59}$ T. A. Pascal and W. A. Goddard III, "Interfacial thermodynamics of water and six other liquid solvents," J. Phys. Chem. B 118(22), 5943-5956 (2014).

${ }^{60} \mathrm{~T}$. Schneider and E. Stoll, "Molecular-dynamics study of a three-dimensional one-component model for distortive phase transitions," Phys. Rev. B 17(3), 1302 (1978).

${ }^{61}$ D. R. Lide and G. W. A. Milne, CRC Handbook of Data on Organic Compounds (CRC Press, 1993).

${ }^{62}$ P. F. Pagoria, G. S. Lee, A. R. Mitchell, and R. D. Schmidt, "A review of energetic materials synthesis," Thermochim. Acta 384(1-2), 187-204 (2002).

${ }^{63}$ S. Naserifar, S. Zybin, C.-C. Ye, and W. A. Goddard III, "Prediction of structures and properties of 2, 4, 6-triamino-1, 3, 5-triazine-1,3,5-trioxide (MTO) and 2, 4, 6-trinitro-1, 3, 5-triazine-1, 3, 5-trioxide (MTO3N) green energetic materials from DFT and ReaxFF molecular modeling," J. Mater. Chem. A 4(4), 1264-1276 (2016).

${ }^{64}$ J. Akhavan, The Chemistry of Explosives (Royal Society of Chemistry, 2011).

${ }^{65}$ T. P. Russell, P. J. Miller, G. J. Piermarini, and S. Block, "Pressure/temperature phase diagram of hexanitrohexaazaisowurtzitane," J. Phys. Chem. 97(9), 19931997 (1993).

${ }^{66}$ M. F. Foltz, C. L. Coon, F. Garcia, and A. L. Nichols III, "The thermal stability of the polymorphs of hexanitrohexaazaisowurtzitane, Part I.," Propellants, Explos., Pyrotech. 19(1), 19-25 (1994).
${ }^{67}$ M. F. Foltz, C. L. Coon, F. Garcia, and A. L. Nichols III, "The thermal stability of the polymorphs of hexanitrohexaazaisowurtzitane, Part II," Propellants, Explos., Pyrotech. 19(3), 133-144 (1994).

${ }^{68}$ D. C. Sorescu and B. M. Rice, "Theoretical predictions of energetic molecular crystals at ambient and hydrostatic compression conditions using dispersion corrections to conventional density functionals (DFT-D)," J. Phys. Chem. C 114(14), 6734-6748 (2010).

${ }^{69} \mathrm{~W}$. L. Jorgensen, D. S. Maxwell, and J. Tirado-Rives, "Development and testing of the OPLS all-atom force field on conformational energetics and properties of organic liquids," J. Am. Chem. Soc. 118(45), 11225-11236 (1996).

${ }^{70}$ J. L. Banks, H. S. Beard, Y. Cao, A. E. Cho, W. Damm, R. Farid, A. K. Felts, T. A. Halgren, D. T. Mainz, J. R. Maple et al., "Integrated modeling program, applied chemical theory (IMPACT)," J. Comput. Chem. 26(16), 1752-1780 (2005).

${ }^{71}$ K. J. Bowers, D. E. Chow, H. Xu, R. O. Dror, M. P. Eastwood, B. A. Gregersen, J. L. Klepeis, I. Kolossvary, M. A. Moraes, F. D. Sacerdoti et al., "Scalable algorithms for molecular dynamics simulations on commodity clusters," in Proceedings of the 2006 ACM/IEEE Conference on Supercomputing, SC'06 (IEEE, 2006), p. 43.

${ }^{72}$ V. Sinditskii, V. Y. Egorshev, V. Serushkin, A. Levshenkov, M. Berezin, S. Filatov, and S. Smirnov, "Evaluation of decomposition kinetics of energetic materials in the combustion wave," Thermochim. Acta 496(1-2), 1-12 (2009).

${ }^{73}$ O. V. Dorofeeva and M. A. Suntsova, "Enthalpy of formation of CL-20," Comput. Theor. Chem. 1057, 54-59 (2015).

${ }^{74}$ S. Zeman and M. Krupka, "New aspects of impact reactivity of polynitro compounds, Part III. Impact sensitivity as a function of the imtermolecular interactions," Propellants, Explos., Pyrotech. 28(6), 301-307 (2003).

${ }^{75}$ A. D. MacKerell, Jr., "Empirical force fields for biological macromolecules: Overview and issues," J. Comput. Chem. 25(13), 1584-1604 (2004).

${ }^{76} \mathrm{H}$. Meirovitch, S. Cheluvaraja, and R. P. White, "Methods for calculating the entropy and free energy and their application to problems involving protein flexibility and ligand binding," Curr. Protein Pept. Sci. 10(3), 229-243 (2009).

${ }^{77}$ R. Ditchfield, W. J. Hehre, and J. A. Pople, "Self-consistent molecular-orbital methods. IX. An extended Gaussian-type basis for molecular-orbital studies of organic molecules," J. Chem. Phys. 54(2), 724-728 (1971).

${ }^{78} \mathrm{~W}$. Hehre and J. Pople, "Self-consistent molecular orbital methods. XIII. An extended Gaussian-type basis for boron," J. Chem. Phys. 56(8), 4233-4234 (1972). ${ }^{79}$ Y. Liu, V. S. Bryantsev, M. S. Diallo, and W. A. Goddard III, "PAMAM dendrimers undergo PH responsive conformational changes without swelling," J. Am. Chem. Soc. 131(8), 2798-2799 (2009).

${ }^{80}$ Y. Duan, C. Wu, S. Chowdhury, M. C. Lee, G. Xiong, W. Zhang, R. Yang, P. Cieplak, R. Luo, T. Lee et al., "A point-charge force field for molecular mechanics simulations of proteins based on condensed-phase Quantum mechanical calculations," J. Comput. Chem. 24(16), 1999-2012 (2003).

${ }^{81}$ H. Berendsen, J. Postma, W. van Gunsteren, and A. J. Hermans, Intermolecular Forces (Reidel, Dordrecht Jerusalem, Israel, 1981). 